\title{
Postsynaptic Changes at a Sensory-to-Motoneuron Synapse Contribute to the Developmental Loss of a Reflex Behavior During Insect Metamorphosis
}

\author{
Gwen A. Jacobs ${ }^{a}$ and Janis C. Weeks ${ }^{b}$ \\ Department of Entomological Sciences, University of California, Berkeley, California 94720
}

The larval-pupal transformation of the tobacco hornworm, Manduca sexta, involves the loss of many larval-specific behaviors, including a simple withdrawal reflex of the abdominal prolegs. This reflex is mediated by monosynaptic excitatory connections between afferents that innervate mechanosensory hairs on the proleg [the planta hairs (PHs)] and motoneurons that innervate proleg retractor muscles. In response to hormonal cues during the final days of larval life, the dendritic arbors of proleg retractor motoneurons regress extensively. Intracellular recordings indicate that the synaptic depolarization evoked in proleg motoneurons by electrical stimulation of PH afferents decreases by $78 \%$ during the larval-pupal transformation, over the same time course that the motoneuron dendrites regress. During the same period the number of PH afferents and the extent of their central projections may decrease slightly. To test the relative contributions of developmental changes in the presynaptic afferents and postsynaptic motoneurons to the weakening of the reflex pathway, we generated heterochronic mosaic pupae that retained one larval proleg. The $\mathrm{PH}$ afferents on the retained proleg retained their larval characteristics while their postsynaptic targets, the proleg motoneurons, appeared to regress normally. In the mosaic hemisegments, electrical stimulation of PH afferents evoked only a small synaptic depolarization of the proleg motoneurons, similar in amplitude to that recorded in normally developing pupae; thus, the developmental status of the afferents was irrelevant to the loss of the reflex. These findings suggest that postsynaptic changes, e.g., structural regression of motoneuron dendrites, may cause the weakening of the reflex pathway.

Received July 20, 1989; revised Oct. 27, 1989: accepted Nov. 7, 1989.

This work was supported by USPHS NRSA fellowship NS07882 awarded to G.A.J., and NIH grant NS23208, an NSF Presidential Young Investigator Award, and an Alfred P. Sloan fellowship awarded to J.C.W. We thank Dr. Gerardus Staal of Zoecon Corporation for the gift of methoprene, Dr. Barry Trimmer for providing some input resistance measurements, Dave Sandstrom for sharing data on APR, Dr. Dorothea L. Trimble for assistance with hormone treatments, histology, and illustration, Jennifer Tharp for histological assistance, and Drs. B. H G. Debu, R. B. Levine, J. P. Miller, W. M. Roberts, D. L. Trimble, B. A. Trimmer, and N. J. Tublitz, and D. J. Sandstrom, for helpful comments on the manuscript

Correspondence should be addressed to Dr. Gwen A. Jacobs, Department of Molecular and Cell Biology, Division of Neurobiology, Life Science Addition, Box 195, University of California-Berkeley, Berkeley, CA 94720.

Present address: Department of Molecular and Cell Biology, Division of Neurobiology, Life Science Addition, Box 195, University of California, Berkeley, CA 94720 .

${ }^{b}$ Present address: Institute of Neuroscience, University of Oregon, Eugene, OR 97403.

Copyright $(1) 1990$ Society for Neuroscience $0270-6474 / 90 / 041341-16 \$ 02.00 / 0$
This is the first demonstrated correlation between the hormonally mediated regression of motoneuron dendrites and the loss of a specific behavior during metamorphosis.

One of the striking aspects of insect metamorphosis is the behavioral differences between larvae, pupae, and adults. For instance, in the tobacco hornworm, Manduca sexta, the most common behaviors of the caterpillar are crawling and feeding, the pupa is relatively quiescent, and the adult moth exhibits flight and reproductive behaviors. As the insect enters each new life stage, most of the behaviors of the previous stage disappear and a new behavioral repertoire appears. To accommodate this behavioral plasticity, many embryonic capabilities, such as neurogenesis, programmed neuron death, the growth or regression of neuronal arbors, and the reorganization of synaptic connections, continue throughout life (e.g., Truman, 1983; Levine and Truman, 1985; Booker and Truman, 1987a; Weeks and ErnstUtzschneider, 1989; Weeks and Levine, 1990). In Manduca, these events have been shown to be controlled by the same hormones that control molting, the ecdysteroids (ecdysone and 20-hydroxyecdysone) and juvenile hormone (JH) (Truman and Schwartz, 1984; Weeks and Truman, 1985, 1986a, b; Levine et al., 1986; Booker and Truman, 1987b; Weeks, 1987; Truman and Reiss, 1988; Levine, 1989; Truman, 1989; Weeks and ErnstUtzschneider, 1989).

A number of studies have investigated the neural and endocrine mechanisms that underlie the appearance of new behaviors at metamorphic molts, i.e., the molts from larva to pupa, or pupa to adult (Truman, 1976; Levine and Truman; 1982, 1983; Levine et al., 1986, 1989; Levine, 1989). The gencral finding has been that the circuitry underlying new behaviors is assembled during the previous stage but is not "enabled" until entry into the new stage, typically in response to a hormonal cue. The formation of new behavioral circuits is often correlated with the growth of new neuronal processes. Less attention has been directed toward the mechanisms by which outmoded behaviors are eliminated at the end of a life stage. Each metamorphic molt is associated with a wave of programmed neuronal death (Taylor and Truman, 1974; Truman, 1983; Giebultowicz and Truman, 1984; Weeks and Truman, 1985) that could eliminate the neurons responsible for outmoded behaviors. In addition, many neurons that do not die undergo structural changes. For instance, at the larval-pupal transformation the dendritic arbors of many neurons regress substantially, which might eliminate synaptic connections (Weeks and Truman, 1985; Kent and Levine, 1988c; Truman and Reiss, 1988). Neural circuits might also be dismantled by more subtle 
changes in the electrophysiological properties of neurons that are independent of structural changes.

In this study we have examined how factors such as these contribute to the developmental loss of a simple proleg withdrawal reflex during the larval-pupal transformation of Manduca. The abdominal prolegs, which are the principal locomotory appendages of the larva, bear a dense array of mechanosensory bristles termed the planta hairs (PHs; Weeks and Jacobs, 1987; Peterson and Weeks, 1988). Each hair is innervated by a peripheral sensory neuron that projects into the central nervous system (CNS). Tactile stimulation of the PHs causes the proleg to be withdrawn, due to contraction of proleg retractor muscles. The behavior is mediated by a simple reflex arc with one central synapse: $\mathrm{PH}$ afferents monosynaptically excite the proleg retractor motoneurons, which in turn evoke contraction of the proleg retractor muscles and retraction of the proleg. Parallel polysynaptic pathways that link the afferents and motoneurons also contribute to the reflex (Weeks and Jacobs, 1987; see Results).

Proleg withdrawal behavior is exhibited throughout the larval stage but is lost after the onset of metamorphosis midway through the final larval instar. Changes in the musculature and cuticle of the prolegs during the larval-pupal transformation (Weeks and Truman, 1985; Wolfgang and Riddiford, 1987) might account for this disappearance of proleg movements, even if the withdrawal reflex circuit within the CNS remained functional. However, in the present experiments we found that the strength of the afferent-to-motoneuron synaptic pathway measured in the CNS decreases markedly during the final days of larval life. This alteration could be due to changes in the presynaptic afferents, the postsynaptic motoneurons, or both. A potentially important postsynaptic change is the extensive ecdysteroid-mediated regression of proleg retractor motoneuron dendrites during the larval-pupal transformation (Weeks and Truman, 1985), which might interrupt synaptic connections with the $\mathrm{PH}$ afferents. By using hormonal manipulations to create heterochronic mosaic animals in which the $\mathrm{PH}$ afferents remained larval while proleg motoneurons became pupal, we were able to test the relative contributions of pre- and postsynaptic changes to the developmental loss of the reflex. Our findings that postsynaptic changes appear to be sufficient to eliminate the reflex suggest that the hormonally induced regression of neuronal dendrites, and resultant weakening of synaptic connections, is one mechanism by which outmoded behaviors may be eliminated during metamorphosis. Some of these results have been reported previously in abstract form (Jacobs and Weeks, 1986; Weeks and Jacobs, 1988).

\section{Materials and Methods}

Experimental animals. Larvae of the tobacco hornworm, M. sexta, were reared in individual containers on artificial diet (Bell and Joachim, 1976) at $27^{\circ} \mathrm{C}$ on a $17 \mathrm{~L}: 7 \mathrm{D}$ photoperiod, to promote synchronous development. After wandering they were placed in individual stoppered chambers bored into wooden blocks. Larvae were staged by the following developmental events (see Fig. 1): ecdysis to the 5th larval instar (day L0), wandering (day WO; indicated by pigmentation changes and gut purge), and ecdysis to the pupal stage (day P0). Between days $L 0$, WO, and $P 0$ the insects were staged by days of development; for example, larvae on the second day after wandering were staged as day W2. Animals of both sexes were used for experiments.

Electrophysiological techniques. For isolated ganglion preparations a $\mathrm{CO}_{2}$-anesthetized insect was cut open along the dorsal midline, the gut was removed, and the abdomen was pinned flat. An abdominal ganglion was dissected from the body and all nerves were cut short except for 2 branches of the ventral nerve (VN): the lateral branch (VNL), containing the axons of proleg retractor motoneurons and no sensory axons, and the third branch of the anterior, anterior branch of the VN (VNAAbr3), containing the axons of the PH afferents and no motor axons (Wccks and Truman, 1984b; Weeks and Ernst-Utzschneider, 1989; see Results). Intracellular recordings were made from motoneuron PPR, which innervates the principal planta retractor muscle (PPRM; Weeks and Truman, 1984b). To identify PPR during recordings, PPRM and its cuticular attachments were dissected out of the body and left attached to the ganglion via VNL (see below). For experiments involving only extracellular recordings, ganglia were not desheathed and were bathed in a saline that mimics the blood (Weeks and Truman, 1985). For intracellular recordings, ganglia were briefly enzyme-treated and desheathed (Weeks and Jacobs, 1987), and bathed in a saline that mimicked the extracellular fluid (modified from Miyazaki, 1980; in mM: NaCl 140; $\mathrm{KCl} \mathrm{5;} \mathrm{CaCl}_{2}$ 4; glucose 28; HEPES 5; $\mathrm{pH}$ 7.4). For "high divalent" saline, $\mathrm{MgCl}_{2}$ and $\mathrm{CaCl}_{2}$ concentrations were both increased to $20 \mathrm{mM}$, with the $\mathrm{NaCl}$ concentration reduced to maintain normal osmolarity. During recordings, ganglia were pinned in a Sylgard-coated dish (Dow Corning, Midland, MI) and transilluminated from below through a darkfield condenser.

Differential extracellular recordings of $\mathrm{VN}$ and $\mathrm{VNL}$ activity were made using glass-tipped suction electrodes, and amplified using highgain, AC-coupled preamplifiers (Grass Instrument Co., Quincy, MA). To stimulate VNAAbr3 electrically, the nerve was draped over a pair of bipolar silver hook electrodes, the electrodes were raised above the bath, and the nerve and wires were coated with petroleum jelly. Voltage pulses of $0.1-1.0 \mathrm{msec}$ duration were delivered to the nerve from a Grass S88 stimulator. The voltage level was adjusted to maximally stimulate all of the sensory axons, as monitored by the size of the compound spike recorded en passant in the proximal VN.

Intracellular recordings were made using glass micropipette electrodes filled with $2 \mathrm{M}$ potassium acetate (resistance 15-20 M $\Omega$ ). If PPR was to be stained, it was reimpaled later with a cobalt electrode (see below). Motoneuron somata were impaled under visual guidance. To identify PPR unequivocally during intracellular recordings, a portion of a PPRM muscle fiber was drawn into a suction electrode and muscle potentials were recorded as described above for nerve recordings. PPK was identified by the occurrence of muscle potentials in PPRM that were timelocked to the motoneuron spikes. As described previously (Weeks and Truman, 1985), muscle potentials can be recorded even during the late stages of muscle of degeneration, so PPR was identifiable by this technique even on day PO. Intracellular recordings were amplified using a Dagan 8800 Total Clamp electrometer (Dagan Instruments, Minneapolis, MN). Synaptic responses were recorded in bridge mode. To ensure the accuracy of membrane potential measurements made while passing current through the recording electrode, the recording was switched periodically from bridge mode to switched current clamp mode for a short period of time, and the electrode capacitance and bridge balance were adjusted. PPR's input resistance was measured by injecting hyperpolarizing current pulses ( 0 to $-3 \mathrm{nA}, 500 \mathrm{msec}$ duration) into the soma, and measuring the steady-state voltage in switched current clamp mode. Input resistance was calculated as the slope of the regressed line through the $\mathrm{I} / \mathrm{V}$ relationship.

Data from experiments that employed only extracellular electrodes were recorded on magnetic tape at $33 / 4 \mathrm{in} . / \mathrm{sec}$ (Vetter Instruments, Rebersberg, PA). Data were played back at $15 / 16$ or $17 / 8$ in./sec onto a pen recorder (Gould Inc., Cleveland, $\mathrm{OH}$ ) for analysis. Data from experiments involving intracellular recordings were recorded and analyzed with a computer-based data system (RC Electronics, Santa Barbara, $\mathrm{CA}$ ). Individual records were displayed on an X-Y plotter.

Histological techniques. Sensory neurons and motoneurons were visualized using conventional cobalt staining techniques. Motoneurons were impaled with electrodes containing $100 \mathrm{~mm}$ hexamine cobaltic chloride (Brogan and Pitman, 1981) and $500 \mathrm{msec}$ current pulses of $10-40 \mathrm{nA}$ were injected at $1 \mathrm{~Hz}$ for $5-10 \mathrm{~min}$. To visualize the central arbors of multiple proleg motoneurons including PPR (see below), VNL was backfilled with $2 \% \mathrm{CoCl}_{2}$. The central arbors of individual $\mathrm{PH}$ afferents were stained by introducing $2 \% \mathrm{CoCl}_{2}$ via the cut hair shaft (Peterson and Weeks, 1988). The combined central termination of the $\mathrm{PH}$ afferents was stained by filling VNAAbr3 with $2 \% \mathrm{CoCl}_{2}$ toward the ganglion. $\mathrm{PH}$ afferent neurons located in the proleg were stained by dissecting out the distal proleg and introducing $2 \% \mathrm{CoCl}_{2}$ via the $\mathrm{VN}$. After cobalt filling, all material was precipitated in saline saturated with $\mathrm{H}_{2} \mathrm{~S}$ gas, fixed, silver-intensified using a modification of the technique 
A

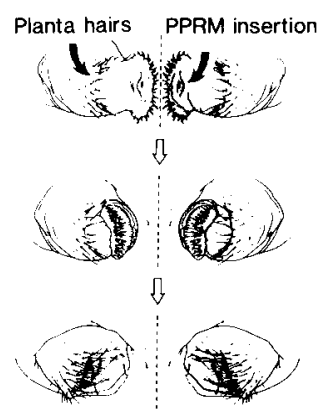

B

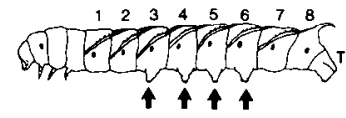

Days of development
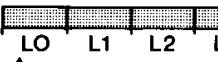

$\uparrow$

Larval ecdysis
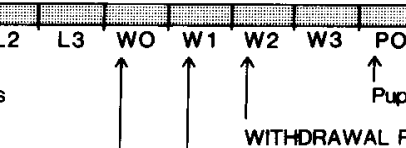

WITHDRAWAL REFLEX ABSENT

PPRM BEGINS DEGENERATION PPR BEGINS REGRESSION

Wandering behavior,

followed by quiescence
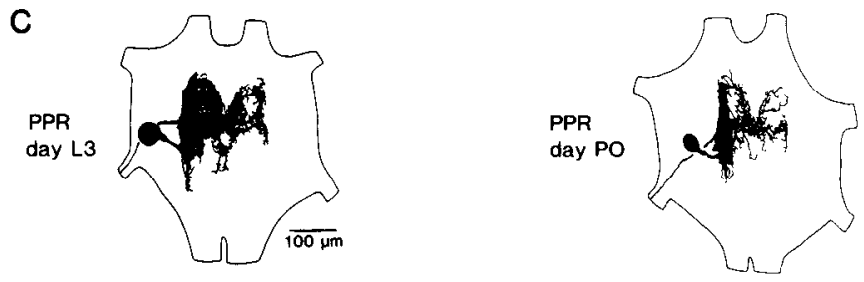

Figure 1. Changes in the proleg neuromuscular system during the larval-pupal transformation. A, The larval proleg withdrawal reflex. A ventral vicw of a segmental pair of prolegs is shown, with a dashed line indicating the ventral midlinc of the body. The planta hair (PH) array and PPRM's ventral insertion point in the planta are indicated in the first panel. PPRM's dorsal insertion point occurs near the spiracle on the lateral body wall (not shown; Weeks and Truman, 1984b). The crochets, sclerotized hooks used for attachment to the substrate, nearly meet at the ventral midline. Tactile stimulation of the PHs evokes proleg retraction as indicated in the successive panels connected by open arrows. The first event is retraction of the crochets mediated by PPRM's contraction (middle panel), followed by retraction of the entire proleg (bottom panel). $B$, Time line of events between ecdysis to the final larval instar (day L0) to pupal ecdysis (day P0). Wandering behavior occurs on day W0. Events relevant to the loss of the proleg withdrawal reflex are listed below the time line. The insets above the time line show a larva and pupa. Abdominal segments $A 1-A 8$, and the terminal segment $(T)$, are indicated on the larva. The abdominal prolegs included in this study are indicated by arrows. $C$, Dendritic regression in PPR. PPR was stained by intracellular iontophoresis of cobalt on days L3 and P0. The outlines of the abdominal ganglia are shown; camera lucida drawings were made in dorsal view; anterior is up. On day P0, PPR's dendritic arbor is much less extensive than in the premetamorphic larval stage.

of Bacon and Altman (1977), and mounted in Canada balsam. Ganglia or proleg tissue were examined in whole mount.

Morphometric measurements. Two morphometric techniques were used. The first technique used VNL backfills, which stain the central processes of 7 motoneurons (Weeks and Truman, 1984b, 1985; Weeks and Ernst-Utzschneider, 1989). All of the motoneurons have dorsal, ipsilateral processes but PPR has, in addition, a dorsal, contralateral arbor which can be viewed in isolation in the contralateral hemineuropil. Camera lucida drawings were made of those VNL backfills which showed excellent resolution of neuronal detail, using a drawing tube on a compound microscope at a final magnification of $520 \times$. A "dendritic density" score was measured for each drawing, which reflected the proportion of the hemineuropil contralateral to the filled nerve that contained PPR's dendritic processes (Weeks and Truman, 1985). A different technique was used to examine the structure of PPR's ventral, ipsilateral arbor. This portion of the arbor was viewed in a compound microscope at a magnification of $100 \times$, and reconstructed with a PC-based computer reconstruction system (Nevin et al., 1988). The number of branch points in the arbor was calculated by the program.

Heterochronic mosaics. Metamorphosis is controlled by the blood titers of the ecdysteroids and JH. The endocrine cues for the larvalpupal transformation are, first, a decrease in the blood JH titer; second, a small elevation of ecdysteroids in the absence of $\mathrm{JH}$ (the commitment pulse, which triggers wandering behavior); and third, a larger rise in ecdysteroids with a low level of JH (the prepupal peak; Riddiford, 1985). The commitment pulse commits larval tissues to subsequent pupal differentiation, with the pupal phenotype being expressed during the molt-inducing prepupal peak (e.g., Weeks and Truman, 1986b). Exposure of a tissue to $\mathrm{JH}$ during the commitment pulse blocks pupal commitment, resulting in the production of larval rather than pupal structures during the prepupal peak.

To produce pupae that retained a larval proleg, the proleg epidermis was treated with a juvenile hormone analog (methoprene, a gift from Zoecon Corp., Palo Alto, CA) during the commitment pulse. Methoprene was dissolved in soft dental wax at $1 \mu \mathrm{g} / \mathrm{ml}$ and frozen in small aliquots until use, at which time it was heated gently and applied to the proleg (see Levine et al., 1986). A thin layer of rubber cement was applied over the treated proleg to help hold the wax in place. Typically, a volume of about $5 \mu \mathrm{l}$ of wax was used to coat the proleg. Fifth instar larvae that weighed between 6.5 and $7.0 \mathrm{gm}$ were treated late in the afternoon on day $\mathrm{L} 2$ and the wax was removed on day $\mathrm{W} 0$. The area of larval epidermis that was retained into the pupal stage varied; some pupae had only a small nub of larval tissue where the proleg had been, while others bore a full-sized proleg. The criteria used to select mosaic pupae for experiments are described in Results. Mosaic animals were used for electrophysiological or anatomical studies on the day of pupal ecdysis (day P0); the same number of days elapsed between wandering and pupal ecdysis in the wax-treated and control animals.

\section{Results}

Loss of the proleg withdrawal behavior during the larval-pupal transformation

Prolegs are present on the third through sixth abdominal segments (A3-A6) of the Manduca caterpillar (Fig. 1B); the anal prolegs were not included in this study. The tip of each proleg, the planta, bears a dense array of mechanosensory hairs designated the planta hairs (Fig. 1 $A$; Peterson and Weeks, 1988). When touched, the PHs evoke a withdrawal reflex in which the stimulated proleg is retracted toward the body (Weeks and Jacobs, 1987). Gentle stimulation of the PHs evokes a strictly ipsilateral withdrawal whereas stronger stimuli cause both prolegs in the segment to retract (Fig. 1A). Proleg withdrawal is caused by the contraction of proleg retractor muscles, including the PPRM (Weeks and Truman, 1984b; Weeks and Ernst-Utzschneider, 1989). PPRM inserts in the distal tip of the planta (Fig. 1A) and its contraction disengages the crochets (sclerotized 
A.

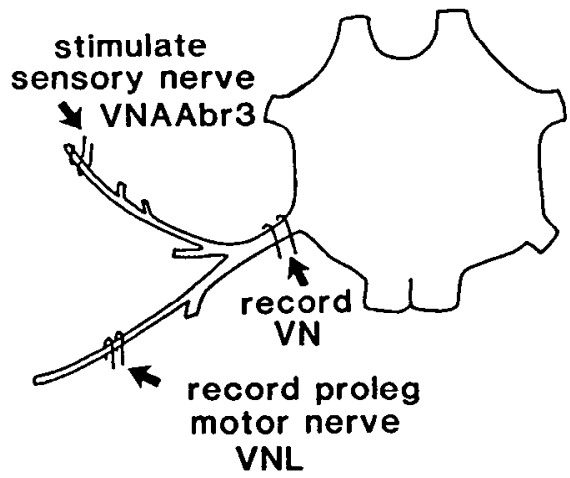

B.
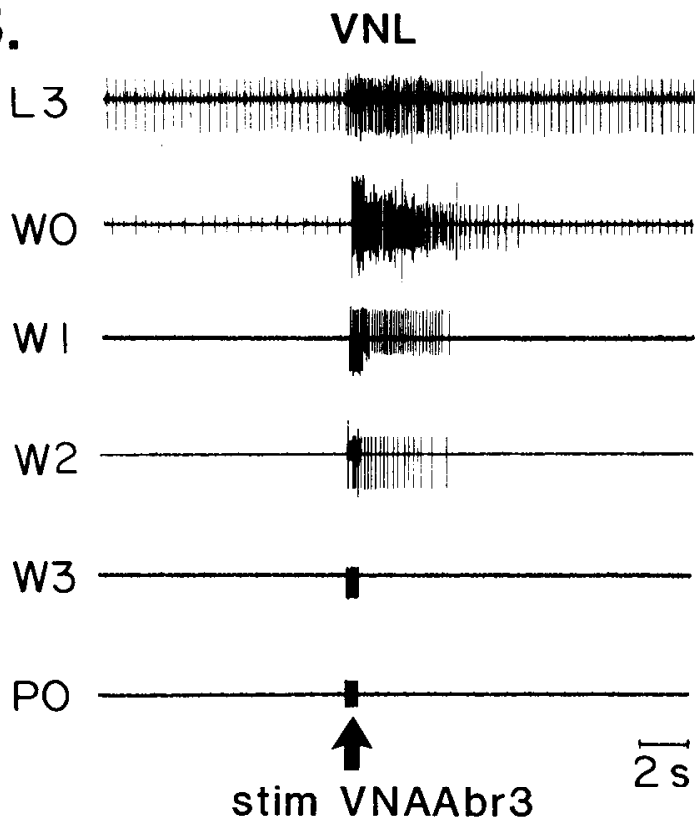

Figure 2. Developmental weakening of the afferent-to-motoneuron pathway. $A$, Recording configuration. In an isolated ganglion preparation, proleg motoneuron spikes were recorded from nerve VNL, while $\mathrm{PH}$ afferents in nerve VNAAbr3 were stimulated with current pulses delivered at $30 \mathrm{~Hz}$ for $500 \mathrm{msec}$. The proximal $\mathrm{VN}$ was monitored en passant to ensure that each stimulus delivered to VNAAbr 3 maximally stimulated all of the $\mathrm{PH}$ afferents, as indicated by the size of their compound action potential in the VN. $B$, Afferent-evoked motor activity. Recordings were made on each of the days of development indicated on the left, in the configuration shown in $A$. The filled arrow at the bottom of the panel indicates the occurrence of the VNAAbr3 stimulus. The ability of electrical stimulation of the PH afferent axons to evoke proleg motoneuron firing declines during the larval-pupal transformation.

hooks on the planta) from the substrate, and withdraws the planta tip. Other muscles retract the more proximal parts of the proleg. PPRM's contractions can be monitored by observing movements of its insertion point in the planta tip. PPRM contracts in response to $\mathrm{PH}$ stimulation with a lower threshold than any of the other retractor muscles, presumably to ensure that the crochets are disengaged before the proleg is withdrawn (Weeks and Jacobs, 1987).

PPRM is singly innervated by motoneuron PPR (Weeks and
Truman, 1984b). PH afferents produce monosynaptic excitatory postsynaptic potentials (EPSPs) in PPR and a subset of other retractor motoneurons (Weeks and Jacobs, 1987; Trimmer and Weeks, 1989). The size of the EPSP produced in a motoneuron by an afferent is correlated with the position of the $\mathrm{PH}$ on the prolcg (Wecks and Jacobs, 1987; Lcvinc and Wecks, 1989), an arrangement that may relate to the somatotopic arrangement of afferent arbors within the CNS (Peterson and Weeks, 1988). Monosynaptic EPSPs from PH afferents have been recorded only in ipsilateral motoneurons (i.e., the motoneurons innervating muscles in the stimulated proleg). A major neural component of the ipsilateral withdrawal reflex is thus the monosynaptic excitation of proleg retractor motoneurons by the $\mathrm{PH}$ afferents. In addition, polysynaptic pathways also link $\mathrm{PH}$ afferents with the ipsi-and contralateral motoneurons (Weeks and Jacobs, 1987; see below).

The proleg withdrawal reflex is present throughout all larval instars but is lost during the larval-pupal transformation. Figure $1 B$ shows a timeline of the 5 th (final) larval instar indicating events relevant to the proleg system. On day W0 the larva exhibits wandering behavior, culminating in the construction of an underground pupation chamber. By day W1 the insect becomes relatively quiescent, exhibiting minimal spontaneous movements but responding if disturbed. The proleg withdrawal reflex can be elicited by stroking the PHs on day $\mathrm{Wl}$, but beginning on day W2 the behavior can no longer be evoked.

Several factors might explain the disappearance of the proleg withdrawal behavior. First, the larval cuticle stiffens markedly after wandering (Wolfgang and Riddiford, 1987). The prolegs flatten toward the body as they stiffen, which might prevent the retractor muscles from causing visible movements. Second, the planta retractor muscles, including PPRM, begin to degenerate after wandering (Fig. $1 B$; Weeks and Truman, 1985). Thus, even if the proleg retractor motoneurons fired, the degenerating muscles might be unable to retract the proleg. Third, the reflex might fail because the PH afferents become nonfunctional or die. Finally, even if the PH afferents continued to operate normally, the reflex could be lost if the synapses between $\mathrm{PH}$ afferents and motoneurons weakened. One possible cause of such weakening is the severe dendritic regression shown by PPR during the prepupal period (Fig. 1C). The extent of PPR's arbor decreases by approximately $40 \%$ between days L3 and P0 (Weeks and Truman, 1985), which might be expected to weaken preexisting synaptic connections. The experiments described below were designed to determine the relative contributions of these factors to the developmental loss of the withdrawal reflex.

\section{Weakening of the afferent-to-motoneuron pathway in isolated ganglia}

To eliminate the possible contributions of changes in the periphery to the loss of the reflex, we turned to an isolated ganglion preparation in which the afferent-to-motoneuron pathway could be tested directly. The axons of proleg motoneurons and $\mathrm{PH}$ afferents run in different subbranches of the $\mathrm{VN}$ : the motoneuron axons run in the VNL; Weeks and Truman, 1984b), while the $\mathrm{PH}$ afferent axons run in a subbranch designated VNAAbr3 (Fig. 2A). To test the efficacy of the afferent-to-motoneuron pathway, we electrically stimulated the $\mathrm{PH}$ sensory nerve while recording the evoked proleg motoneuron activity in VNL on days of development spanning the larval-pupal transformation. An en passant electrode placed on the proximal VN was monitored continuously in all experiments to ensure that the 
VNAAbr3 stimulus maximally activated the $\mathrm{PH}$ afferent axons. As shown in Figure $2 B$, a brief train of stimuli applied to the PH sensory nerve in larvae (day L3) evoked a burst of motor activity in the proleg motor nerve that outlasted the stimulus. The motor activity consisted of PPR's spikes, which are among the largest in the VNL (Trimmer and Wecks, 1989; data not shown), and those of other motoneurons with smaller spikes. This motor response is similar to that seen when the PHs are stroked in semi-intact preparations (Weeks and Jacobs, 1987) and constitutes the neural correlate of the proleg withdrawal reflex. After wandering the response weakened. The reflex was present in all ganglia tested on day W0 $(n=3)$ and day W1 $(n$ $=3$ ), but on day $W 2$ only 1 of 3 ganglia tested produced an afferent-evoked motor burst (this example is shown in Fig. 2B). In the other 2 ganglia, an occasional spike was evoked during the stimulus train but none occurred after the stimulus ended. On day $\mathrm{W} 3(n=3)$ and day $\mathrm{P} 0(n=3)$, sensory stimulation did not evoke motor spikes, even during the stimulus train. These experiments indicate that the afferent-to-motoneuron pathway weakens during the final days of larval life and that by pupation $\mathrm{PH}$ afferent stimulation is no longer able to bring proleg motoneurons to spike threshold.

To further examine this decrease in afferent-evoked excitation, we delivered single shocks to VNAAbr3 while recording intracellularly from PPR, on days L3 and P0 (Fig. 3A). PPR's resting potential hyperpolarizes somewhat over this period of development (Table 1, A, B), so the motoneuron was held at a series of membrane potentials in each experiment to allow synaptic responses to be compared at the 2 stages. Figure $3 B$ shows typical recordings made on days L3 and P0. When PPR was held at $-50 \mathrm{mV}$ on day $\mathrm{L} 3$, the afferent stimulus evoked an abrupt depolarization which triggered action potentials, followed by a hyperpolarization. As PPR's membrane potential was increased to -60 and $-70 \mathrm{mV}$, the depolarizing component increased in height, the number of evoked spikes decreased (because the neuron was held farther from spike threshold), and the hyperpolarizing component reversed. The depolarization presumably resulted from mono- and polysynaptic excitatory inputs, and the hyperpolarization from polysynaptic inhibition (Weeks and Jacobs, 1987).

On day P0 (Fig. 3B), the evoked response was markedly smaller and was always subthreshold for action potential production; this was not due to an inability of PPR to produce action potentials, because the motoneuron fired normally when depolarized by injected current, or when depolarizing current was combined with afferent stimulation (data not shown). The synaptic response on day $\mathrm{P} 0$ was monophasic at membrane potentials between -50 and $-70 \mathrm{mV}$, consisting of a depolarization that

Figure 3. Developmental decrease in the size of the afferent-evoked depolarization in PPK. $A$, Kecording configuration. Intracellular recordings were made from PPR in an isolated, desheathed ganglion. PPR was identified by the occurrence of potentials recorded in PPRM that were time-locked to the motoneuron spikes. The stimulation and monitoring of $\mathrm{PH}$ afferent activity was the same as in Figure 2. $B$, Synaptic potentials recorded in normal saline. On days L3 (left) and PO (right), PPR was held at a series of holding potentials $(-50,-60$, and -70 $\mathrm{mV}$ ) while delivering a single supramaximal shock to VNAAbr3 (arrows). Two superimposed sweeps are shown in each case. $C$, Effects of high divalent saline. On days $\mathrm{L} 3$ (left) and P0 (right), the afferent-evoked synaptic depolarization was recorded in normal saline (top traces), after equilibration of the response in high divalent $\left(20 \mathrm{mM} \mathrm{Ca}^{2+}, 20 \mathrm{~mm}^{2+}\right)$ saline (middle trace), and after recovery in normal saline (bottom trace).
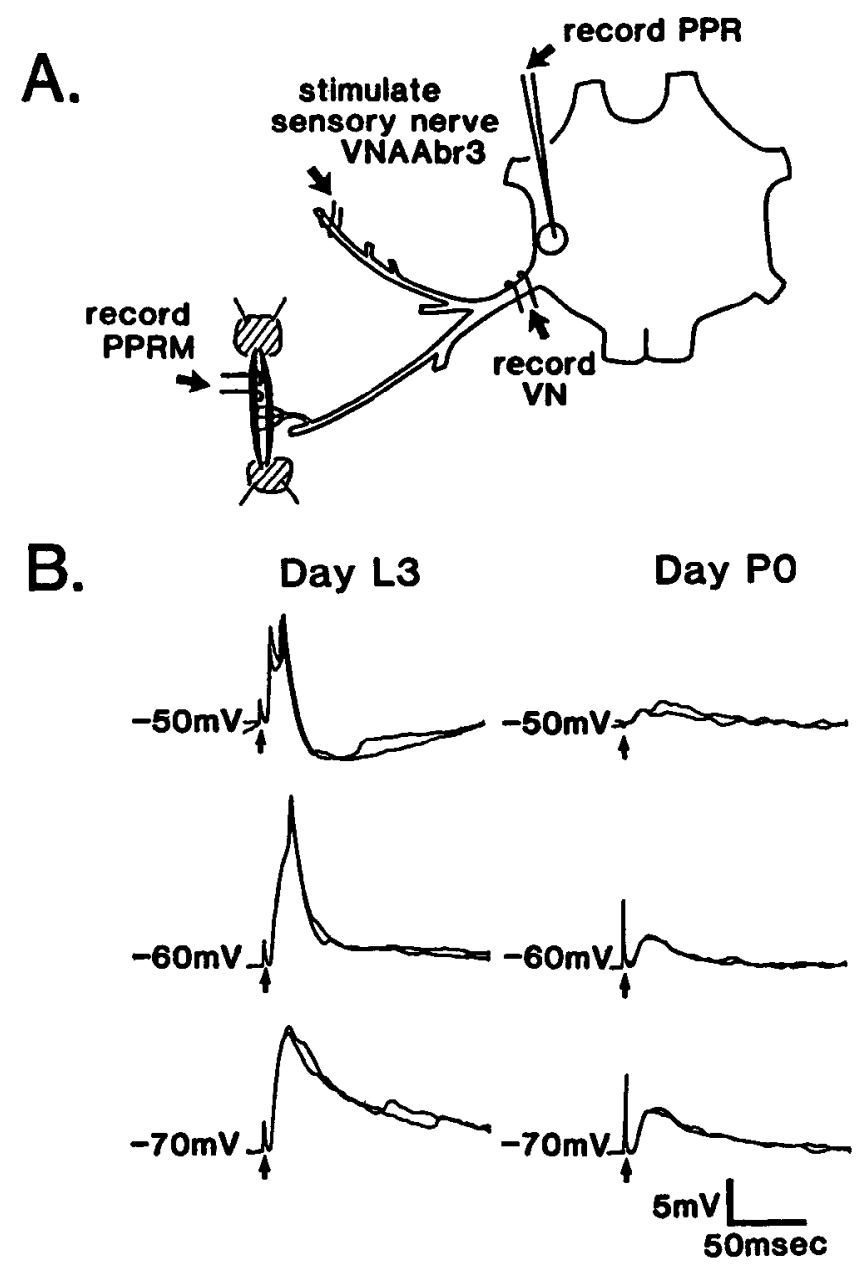

Q.

Day L3 Day PO

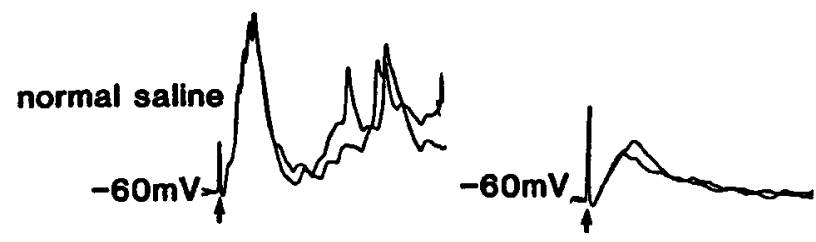

high divalent
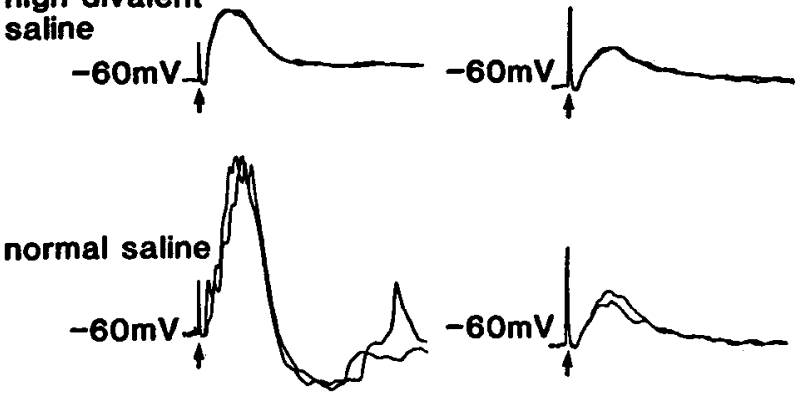

PPR was held at $-60 \mathrm{mV}$ during all trials; arrows mark stimulus artifacts. Two superimposed sweeps are shown in each case. High divalent saline, which reduces or eliminates polysynaptic components, produced a reversible decrease in the amplitude of the afferent-evoked depolarization (in the examples shown, high divalent saline caused a reversible decrease of approximately $50 \%$ on day L3 and $20 \%$ on day P0). Even in high divalent saline, the amplitude of the response decreased between days $\mathrm{L} 3$ and $\mathrm{P} 0$, just as in normal saline (compare the middle traces; see Table 1). The scale bar in $B$ refers to both panels. 
Table 1. Comparison of electrophysiological properties of PPR on day L3, day P0, and in heterochronic mosaic hemisegments on day P0

\begin{tabular}{|c|c|c|c|}
\hline & $\begin{array}{l}\text { A } \\
\text { Day L3 }\end{array}$ & $\begin{array}{l}\text { B } \\
\text { Day P0 }\end{array}$ & $\begin{array}{l}\text { C } \\
\text { Mosaic } \\
\end{array}$ \\
\hline \multicolumn{4}{|l|}{ Normal saline } \\
\hline Resting potential $(\mathrm{mV} \pm \mathrm{SEM}, n)$ & $50.5 \pm 1.4(4)$ & $56.5 \pm 2.1(10)$ & $53.7 \pm 2.3(6)$ \\
\hline Afferent-evoked depolarization at $-60 \mathrm{mV}(\mathrm{mV} \pm \mathrm{SEM}, n)$ & $16.6 \pm 1.6(4)$ & $3.7 \pm 1.0(6)^{a}$ & $3.0 \pm 1.1(6)^{b}$ \\
\hline Input resistance $(\mathrm{M} \Omega \pm \mathrm{SEM}, n)$ & $13.1 \pm 1.3(9)$ & $21.7 \pm 2.7(9)^{a}$ & $24.1 \pm 3.6(6)^{b}$ \\
\hline \multicolumn{4}{|l|}{ High divalent saline } \\
\hline Resting potential $(\mathrm{mV} \pm \mathrm{SEM}, n)$ & $57.7 \pm 3.9(3)$ & $57.4 \pm 2.0(5)$ & $\mathrm{nt}$ \\
\hline Afferent-evoked depolarization at $-60 \mathrm{mV}(\mathrm{mV} \pm \mathrm{SEM}, n)$ & $8.7 \pm 1.3(3)$ & $2.8 \pm 0.9(4)^{a}$ & $\mathrm{nt}$ \\
\hline
\end{tabular}

${ }^{a}$ Significantly different from corresponding value on day $\mathrm{L} 3(p<0.01)$.

${ }^{b}$ Not significantly different from corresponding value on day PO $(p>0.5)$.

All comparisons are 2-tailed $t$-tests. nt, not tested.

increased in amplitude with increasing membrane potential hyperpolarization (Fig. $3 B$ ). These data indicate that between days L3 and P0 the synaptic depolarization evoked in PPR by stimulating the $\mathrm{PH}$ afferent axons decreased markedly, and a hyperpolarizing component of the response disappeared. To quantify these differences we measured the peak amplitude of the evoked synaptic depolarization (ignoring superimposed action potentials) while holding PPR at $-60 \mathrm{mV}$. As shown in Table $1, \mathrm{~A}, \mathrm{~B}$, the mean amplitude of the depolarization decreased by $78 \%$ between days $\mathrm{L} 3$ and $\mathrm{PO}$.

Because we were most interested in changes in the monosynaptic component of the response, we repeated these experiments in saline containing elevated levels of $\mathrm{Ca}^{2+}$ and $\mathrm{Mg}^{2+}$ ions ("high divalent" saline) to minimize polysynaptic effects (Cohen et al., 1978; Weeks and Jacobs, 1987; Trimmer and Weeks, 1989). Typical records are shown in Figure $3 C$. High divalent saline caused the amplitude of the afferent-evoked depolarization to decrease from its level in normal saline (Fig. $3 C$; Table 1, A, B), presumably by reducing or eliminating polysynaptic pathways. Nevertheless, the mean amplitude of the remaining component measured with PPR held at $-60 \mathrm{mV}$ decreased by $68 \%$ between days $\mathrm{L} 3$ and $\mathrm{P} 0$, similar to the decrease measured in normal saline ( $78 \%$ ). These findings suggest that the observed decrease in the magnitude of the afferent-evoked depolarization of PPR between days L3 and P0 includes a decrease in the monosynaptic component of the reflex.

A factor that influences the interpretation of these data is PPR's input resistance $\left(R_{\text {in }}\right)$. For instance, PPR regresses substantially between days L3 and P0 (Fig. 1C), and the level of background synaptic activity in motoneurons decreases during the prepupal period (Weeks and Truman, 1984a, b), both of which might influence $R_{\text {in }}$. We measured $R_{\text {in }}$ on days L3 and $\mathrm{PO}$ in normal saline by determining the slope of the $\mathrm{I} / \mathrm{V}$ relationship for hyperpolarizing current steps. As indicated in Table 1 , A, B, the PPR's mean $R_{\text {in }}$ exhibited a statistically significant increase of about $40 \%$ between days L3 and PO. This increase in $R_{\text {in }}$ would tend to increase the magnitude of the synaptic response recorded on day $\mathrm{P} 0$, as opposed to the decrease that we observed experimentally. Therefore, the measurements presented here may, in fact, underestimate the magnitude of the differences measured between days $\mathrm{L} 3$ and PO (see Discussion).

We conclude that the larval-pupal transformation is accompanicd by a major decrease in the strength of the afferent-tomotoneuron pathways that mediate the proleg withdrawal reflex, including a decrease specifically in the monosynaptic component. The time course of the weakening and loss of the proleg withdrawal reflex parallels exactly the time course of PPR's regression; both begin after wandering and are complete by pupation (Fig. 2B; Weeks and Truman, 1985).

\section{Possible presynaptic factors involved in the loss of the proleg withdrawal reflex}

The weakening of the monosynaptic component of the reflex could be due to developmental changes in the presynaptic $\mathrm{PH}$ afferents, in the postsynaptic motoneurons, or in both. Possible changes on the presynaptic side include programmed death of the $\mathrm{PH}$ afferents, regression of their axonal arbors, or alterations in synaptic release properties. For instance, independent of changes in PPR, the decrease in the evoked depolarization could result from (1) the death of some of the $\mathrm{PH}$ afferents, with the remainder producing the same size EPSP as in the larva, or (2) no change in the number of afferents synapsing on PPR, but with each producing a smaller EPSP. The following experiments were designed to address such issues.

To determine whether the number of afferents was reduced by cell death between days $\mathrm{L} 3$ and $\mathrm{P} 0$, we visualized and counted $\mathrm{PH}$ afferent cell bodies within the proleg epidermis by retrograde cobalt staining via VNAAbr3. Within larval prolegs (Fig. 4A) the bipolar $\mathrm{PH}$ afferent neurons occurred in an orderly array, with the dendrite of each neuron associated with an external cuticular hair. We counted the number of $\mathrm{PH}$ afferents stained via VNAAbr 3 in segments $A 4$ and $A 5$ and found $43 \pm 2$ (SEM; $n=9$ ) neurons per proleg in 5 th instar larvae. Staining in day P0 animals was carried out a few hours before ecdysis, prior to tanning of the new pupal cuticle. During the larval-pupal transformation the proleg epidermis detaches from the old larval cuticle and flattens toward the body to eliminate the bulge formed previously by the proleg. As shown in Figure $4 B$, this epidermal remodeling moved the $\mathrm{PH}$ afferent neurons much closer together. Their elongated dendrites, which were no longer associated with hairs, terminated in the region of epidermis previously occupied by the hair array. Figure $4 C$ shows an enlarged view of a single $\mathrm{PH}$ afferent on day $\mathrm{P} 0$. We counted $37 \pm 2$ (SEM; $n=7$ ) stained neurons in these preparations, representing a statistically significant decrease of $9 \%$ from the larval count ( $p<0.05,2$-tailed $t$-test). However, the close apposition of the $\mathrm{PH}$ afferent neurons in day $\mathrm{PO}$ animals made accurate counting difficult, so we were not confident that the decrease was not artifactual. Tanning of the new pupal cuticle after ccdysis made visualization of the neurons impossible, so we were not able to count them at later times.

We also examined the central termination field of the $\mathrm{PH}$ 

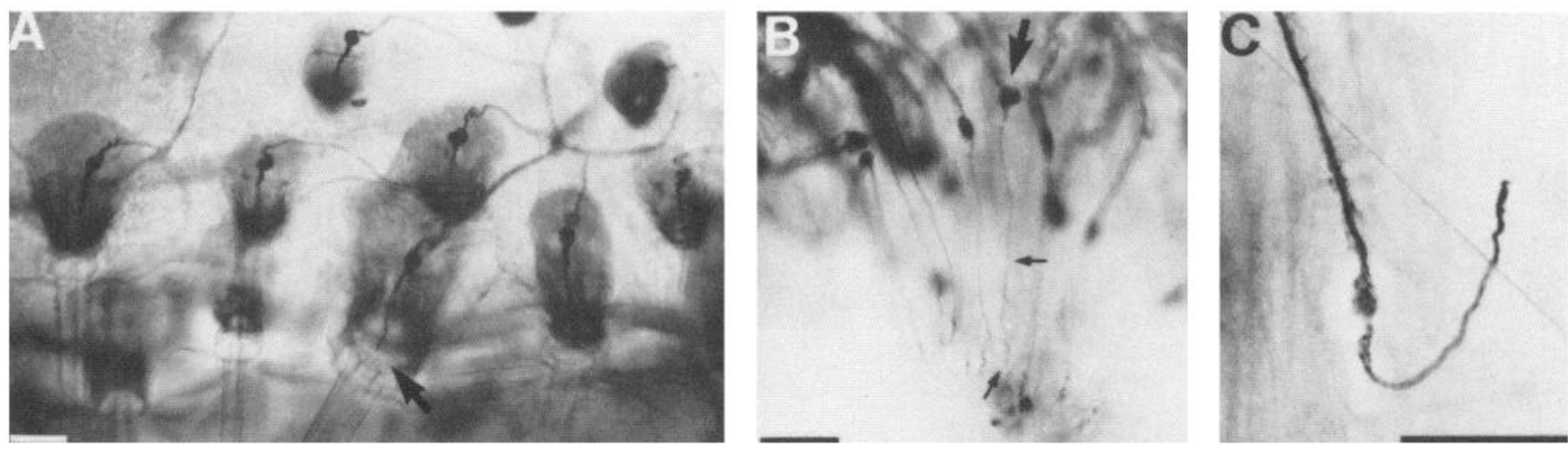

Figure 4. Morphology of $\mathrm{PH}$ afferents in larvae and pupae. $\mathrm{PH}$ afferents in the periphery were visualized by retrograde cobalt staining via VNAAbr3 and photographed in whole mounts of proleg epidermis. The distal (ventral) tip of the proleg is oriented down in $A$ and $B$. $A$, Larval PH afferents. A portion of the $\mathrm{PH}$ array is shown. The dendrite of each afferent is associated with the socket of a planta hair (arrow). The cell bodies lie a small distance from the base of the hair, and the axons of different afferents join together, eventually forming VNAAbr3. The oblong, dark structure surrounding the afferent neuron at the base of each hair consists of the support cells, which darkened during cobalt staining. $B, C$, PH afferents on day P0. The photographs in $B$ and $C$ were taken in the same region as $A$, but a few hours before pupal ecdysis. In $B$, the cell body of a PH afferent is marked by a large arrow, and the middle and end of its dendrite by small arrows. Several cell bodies and their dendrites are apparent. During the larval-pupal transformation the PH afferents become crowded together and they lose their association with hairs. Their dendrites terminate blindly in the epidermis. In $C$, a PH afferent stained on day $\mathrm{P} 0$ is shown at higher magnification. The support cells are visible as a lighter area around the cell body and proximal dendrite. Scale bar in all panels, $50 \mu \mathrm{m}$.

afferents by anterograde cobalt filling from VNAAbr3. Individual PH afferents arborize somatotopically in specific neuropil regions based on their location in the PH array (Peterson and Weeks, 1988), with the VNAAbr3 termination field revealing the morphological extent of their combined arbors. Typical fills are shown in Figure 5. The density of the termination field clearly decreased between day L3 $(n=5)$ and day P2 $(n=6)$ (Fig. 5, $A, C)$; in most fills made on day P0 $(n=5)$ the termination field appeared to be slightly reduced from that on day L3 (Fig. 5B). The decreased size of the PH afferent projection could have been due to the death of a portion of the afferent population, the regression of some or all of the neurons, or a combination of both. A preferable approach would have been to stain individual $\mathrm{PH}$ afferents on day P0 to look directly for structural changes. However, it has not been possible to stain them by the usual technique of introducing cobalt into the hair shaft, presumably because the dendrites are disconnected from the hairs at this time (Fig. 4, $B, C$ ).

These anatomical observations suggest that the number of PH afferent neurons and/or the extent of their central arborizations may decrease somewhat between days $\mathrm{L} 3$ and $\mathrm{P} 0$, during the time that the proleg withdrawal reflex is lost. It is impossible to estimate what effect this might have on afferent-to-motoneuron transmission, and on the proleg withdrawal reflex. Accordingly, to determine the relative contribution of changes in the pre- and postsynaptic neurons, it was necessary to use a different technique.

\section{Heterochronic mosaics}

During the larval-pupal transformation, a small elevation of blood ecdysteroids (the commitment pulse) that occurs in the absence of JH commits tissues to pupal development (see Materials and Methods). Levine et al. (1986) developed a technique to block the pupal commitment of a small patch of epidermal cells by coating the cuticle with wax impregnated with a $\mathrm{JH}$ analog (JHA) during the commitment pulse. This treatment produces a heterochronic mosaic pupa bearing a small patch of larval cuticle and sensory hairs. We used this technique to produce pupae that bore a single larval proleg on segment A5 (Fig. $6 A$ ). During the prepupal period, the JHA-treated proleg epidermis secreted larval cuticle and planta hairs, whereas the rest of the epidermis formed pupal structures and cuticle. Treated
A. Day L3

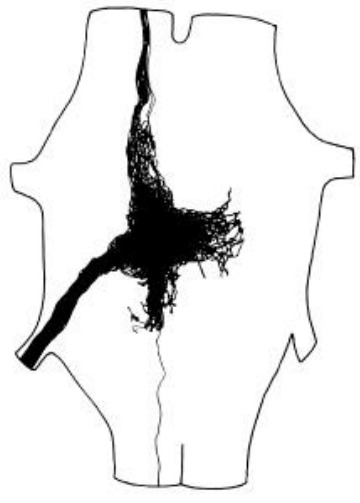

B. Day PO

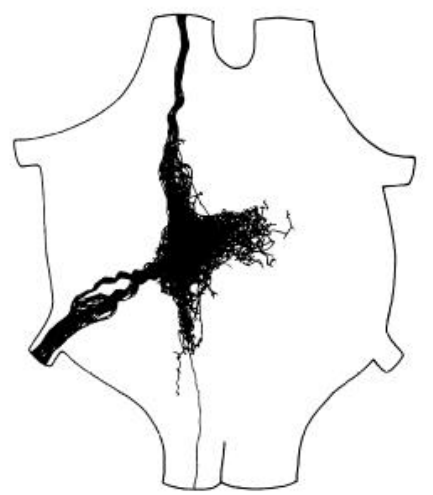

C. Day P2

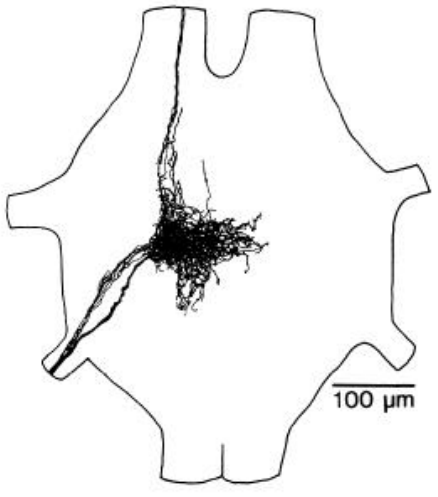

Figure 5. Central projections of $\mathrm{PH}$ afferents. On day $\mathrm{L} 3(A)$, day $\mathrm{P} 0(B)$, and day P2 $(C)$ the central termination field of the $\mathrm{PH}$ afferents was visualized by anterograde cobalt staining via VNAAbr3. Ganglia are oriented as in Figure $1 C$. Afferents enter the ganglion via the $\mathrm{VN}$, arborize within the ipsilateral neuropil, and to a lesser extent in the contralateral neuropil. Intersegmental projections are more extensive anteriorly than posteriorly. Between days $\mathrm{L} 3$ and $\mathrm{P} 2$, the central projection becomes less dense, indicating the regression and/or death of $\mathrm{PH}$ afferents. 

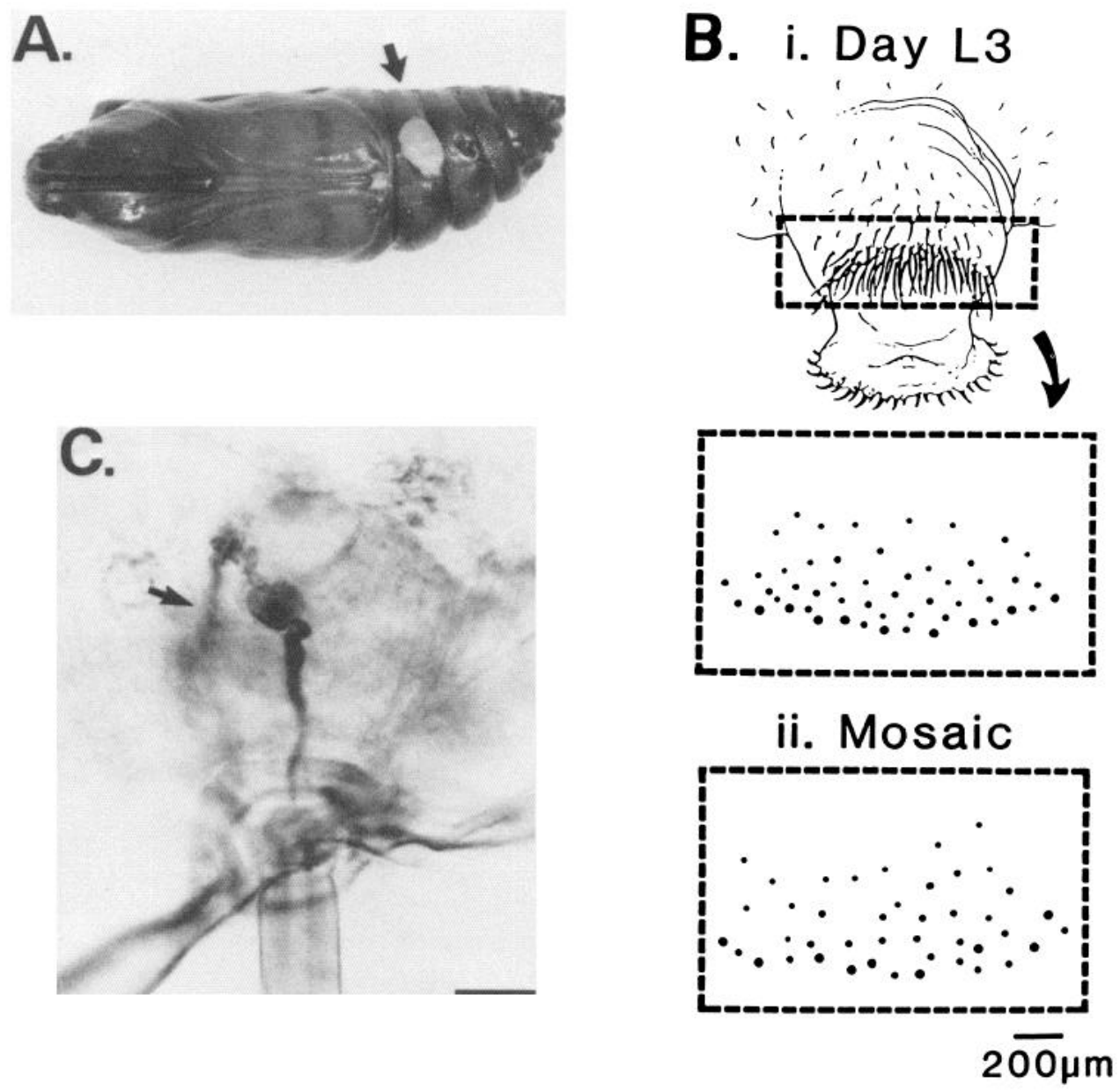

Figure 6. Heterochronic mosaic pupae. A, This pupa photographed on day P1 bears a retained larval proleg on the left side of abdominal segment A5 (arrow). Otherwise, the external morphology appears completely normal. The extent of the retained larval tissue is typical of the animals used for experiments. Anterior is to the left. B. Comparison of PH arrays on the prolegs of larvae and of mosaic pupae. $i$, Day L3. The drawing shows a lateral view of a larval abdominal proleg, with the $\mathrm{PH}$ array outlined by dashed lines. Anterior is to the left. The location and relative diameter of PHs on the proleg of a day L3 larva are shown enlarged in the lower box. A dot represents the position of each PH, with the diameter of each dot reflecting the relative diameter of the hair at its base. As described previously, the largest diameter hairs are located distally on the proleg, and the density and diameter of hairs decrease in the proximal direction (Peterson and Weeks, 1988). The hairs were drawn using a camera lucida attachment on a stereomicroscope. $i i$, PH array on a mosaic pupa. The orientation is the same as in $i$. These hairs were drawn from the retained larval proleg on a heterochronic mosaic pupa, on day P0. The overall arrangement of the hairs is similar to that of a normal larva, including the position dependence of hair diameter and hair density. $C$, Morphology of a larval PH afferent on a mosaic pupa. The photomicrograph shows a $\mathrm{PH}$ afferent and its associated hair, located on a retained proleg of a heterochronic pupa on day P0. The afferent was stained retrogradely with cobalt, and the tissue is oriented as in Figure 4A. As in larvae (Fig. 4A), the dendrite is associated with the base of the hair, the neuron is surrounded by slightly darker staining support cells, and the axon projects to the CNS (the axon leaves the plane of focus at the arrow). Scale bar, $25 \mu \mathrm{m}$.

animals were examined for mosaic characteristics on day $\mathrm{P} 0$, a few hours before pupal ecdysis. Retained prolegs had the bluepigmented epidermis and flexible cuticle characteristic of larvae, while the remainder of the body had pupal cuticle. The proleg retractor muscles degenerated normally in the treated hemisegments, preventing any movements in response to PH stimulation. Most prolegs bore few or no crochets, as was expected given the different hormonal sensitivity of the crochet epidermis (Fain and Riddiford, 1977).

The number and arrangement of PHs on retained prolegs varied. Some prolegs had only a few PHs, whereas others had PH arrays with a similar number and arrangement of hairs as in normal larvae. The mean number of PHs per proleg in segment A5 of normal fifth instar larvae was $49 \pm 4$ (SEM; $n=$ 6). Among mosaic animals, only those with a $\mathrm{PH}$ array containing at least 36 hairs ( $75 \%$ of the control number) were used for experiments. As illustrated in Figure $6 B$, the arrangement of PHs on retained prolegs resembled that of normal larvae. The photomicrograph in Figure $6 \mathrm{C}$ shows the morphology of a $\mathrm{PH}$ afferent within the epidermis of a retained proleg, stained on day P0. The structure of the PH afferent neurons in mosaic animals, and the anatomical relationship of their dendrites with $\mathrm{PH}$ hairs, were indistinguishable from those of normal larvae (Fig. 4A).

The objective of the JHA treatments was to produce animals in which the $\mathrm{PH}$ afferents on the retained proleg remained in the larval state while the CNS, including PPR, became pupal. Tests of whether the proleg withdrawal reflex was retained or lost in the mosaic hemisegment would then indicate whether developmental changes in the $\mathrm{PH}$ afferents were necessary for the normal loss of the reflex. Previous studies by Levine and collaborators (Levine et al., 1986; Levine, 1989) showed that 
A

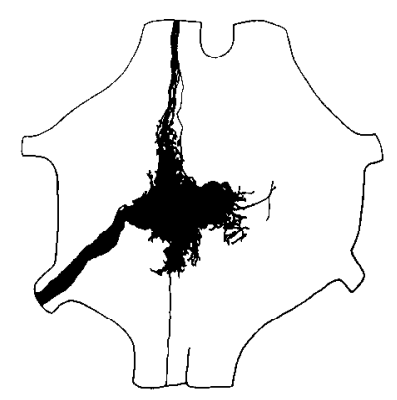

B

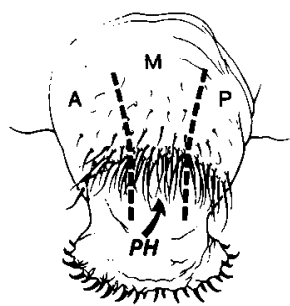

C

Day L3
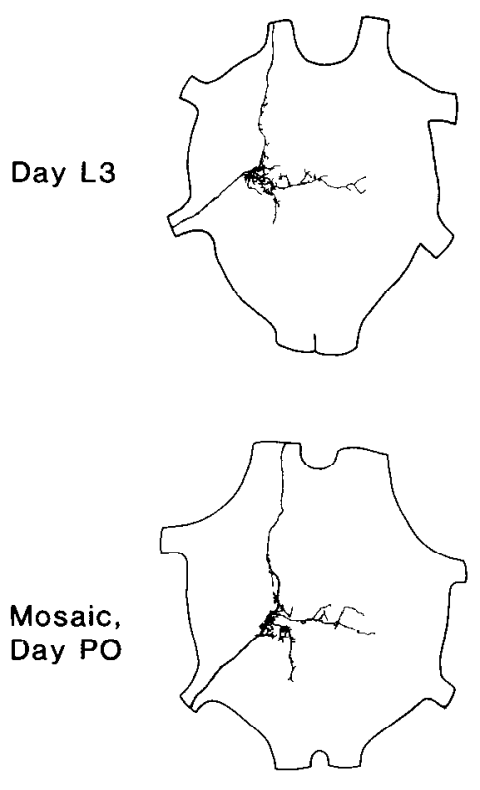

Middle
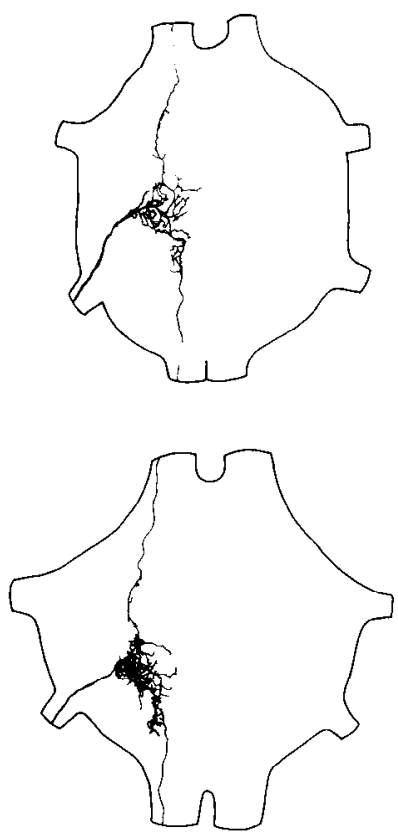

Posterior
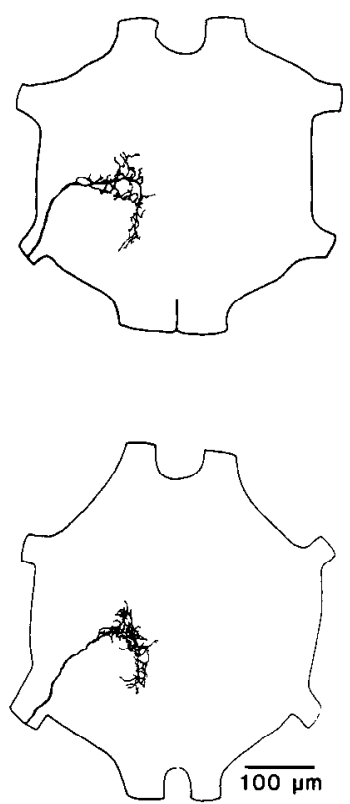

Figure 7. Comparison of central projections of PH afferents in larvae and heterochronic mosaics. A, Anterograde cobalt staining of VNAAbr3 from a retained larval proleg of a heterochronic mosaic pupa on day P0. The extent of the central termination field appears similar to that of normal larvae (Fig. 5A). B, For cobalt staining of individual afferents, the $\mathbf{P H}$ array was divided into anterior $(A)$, middle $(M)$, and posterior $(P)$ regions (Peterson and Weeks, 1988). $C$, Central arborizations of individual PH afferents located in the anterior, middle, and posterior regions of the proleg, stained on day L3 in normal larvae (top row), and on day P0 in heterochronic mosaic pupae (bottom row). The anatomy of the afferents on retained larval prolegs is similar to that of normal larvae. Ganglia are oriented as in previous figures. The scale bar refers to pancls $A$ and $C$.

neurons in heterochronic mosaics do express the phenotype appropriate for the hormonal environment of the neuron's cell body. However, it was necessary to confirm this result in our mosaics.

\section{Central projections of $\mathrm{PH}$ afferents in heterochronic mosaics}

We stained the central termination field of the PH afferents of retained prolegs by filling VNAAbr3 (Fig. $7 A ; n=9$ ). In retained prolegs with full $\mathrm{PH}$ arrays, the field resembled that of normal larvae (compare Figs. $7 A$ and $5 A$ ). In retained prolegs that bore fewer PHs, the termination field was sparser (not shown). In addition, individual $\mathrm{PH}$ afferents on retained larval prolegs were stained with cobalt $(n=22)$ to compare their central arborizations with those of larval controls. In previous studies of normal larvae the $\mathrm{PH}$ array was divided into anterior, middle, and posterior regions (Fig. $7 B$; Peterson and Weeks, 1988). The axonal terminations of afferents from the 3 regions map somatotopically within the ganglionic neuropil. Figure $7 C$ shows

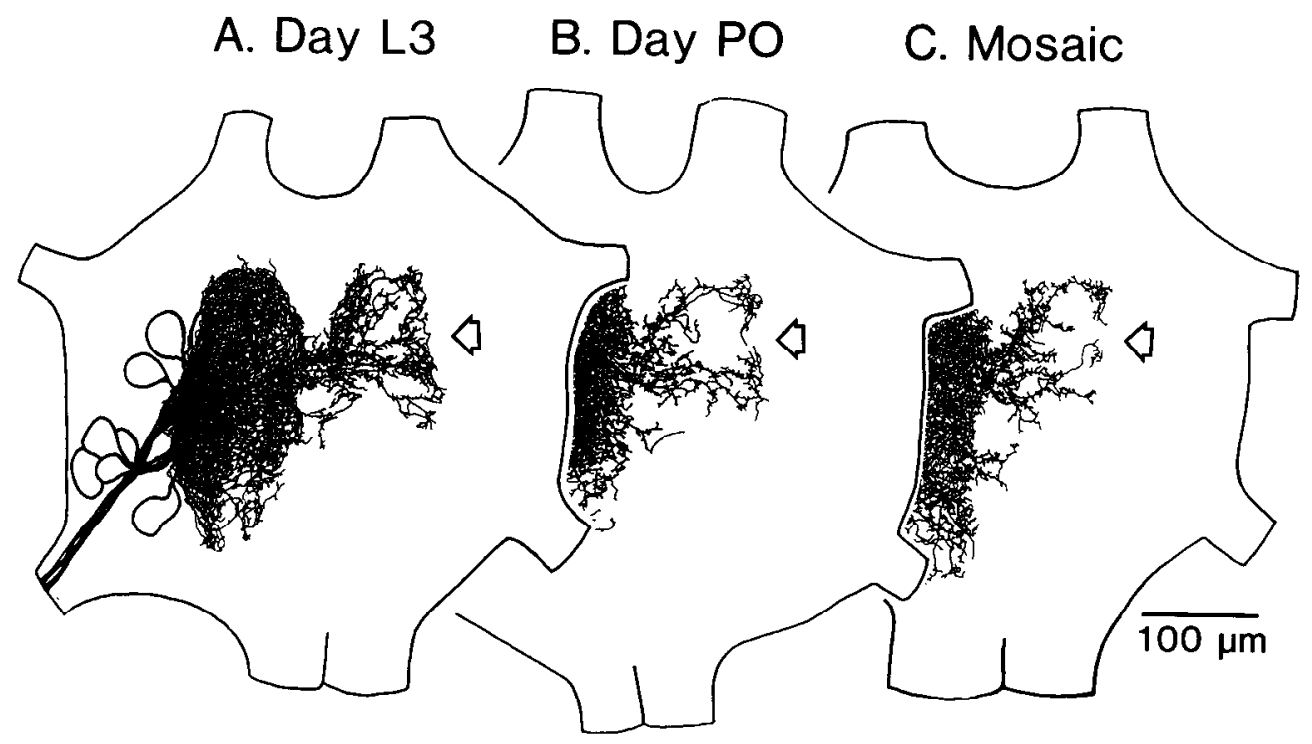

Figure 8. Dendritic regression of PPR. Cobalt backfills of nerve VNL, which carries the axons of 7 proleg motoneurons, were made on day $\mathrm{L} 3(A)$, day P0 $(B)$, and in mosaic hemisegments of heterochronic mosaic pupac on day $\mathrm{P} 0$ (C). The 7 motoneuron cell bodies stained via $\mathrm{VNL}$ are shown in outline in $A$. PPR is the only motoneuron that projects contralaterally, and its contralateral arbor (arrows) is therefore visible in isolation in the VNL backfills. PPR's contralateral arbor regresses substantially between day $\mathrm{L} 3$ and day $\mathrm{P} 0$ (compare $A$ and $B$ ). In heterochronic mosaics stained on day $\mathrm{PO}(C)$, regression of the contralateral arbor of the PPR associated with the retained larval proleg is similar to that of normal day P0 pupae (compare $B$ and $C$; see also Table 2). The individual dendritic density scores for the 3 backfills are: $A, 0.40$ $B, 0.26 ; C, 0.21$. 
A.

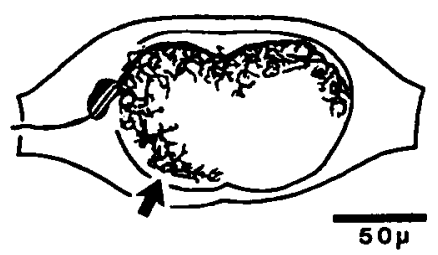

B.

$$
\text { Day L3 }
$$

$$
\text { Day PO }
$$
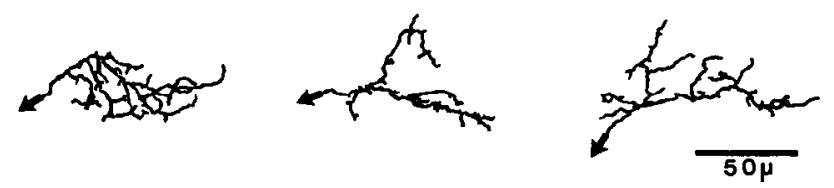

C.

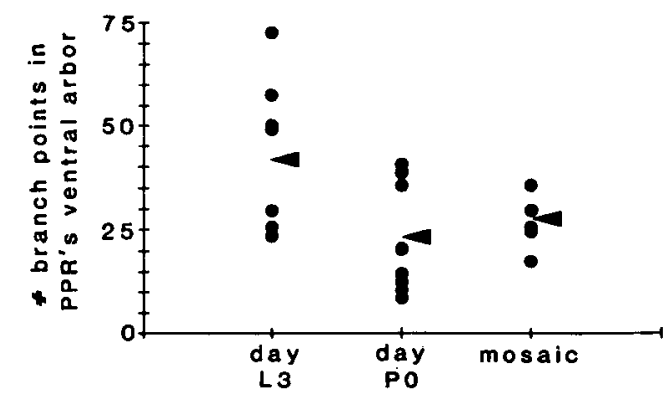

Figure 9. Regression of PPR's ventral arbor. A, The drawing shows PPR's structure in transverse view (dorsal is up), with the arrow indicating the ventral, ipsilateral arbor. The dorsal, contralateral arbor is also apparent. $B$. Drawings of PPR's ventral arbor on day L3, day P0, and in a heterochronic mosaic hemisegment on day P0. The drawings were made in ventral view; arrows indicate where the main process of the ventral arbor joins the rest of the arbor. The ventral arbor is less complex on day P0 and in mosaics, as compared to normal larvae. $C$, Comparison of the number of branch points in PPR's ventral arbor on day L3, day P0, and in heterochronic mosaic hemisegments on day P0. The number of branch points was counted in computer reconstructions of cobalt-filled material in ventral view, such as is shown in $B$. The mean number of branch points ( \pm SEM, $n$; indicated by arrowheads) was: day L3, $40.1 \pm 8.0$ (7); day $\mathrm{P} 0,22.6 \pm 5.1$ (8); mosaic, $25.5 \pm$ $3.6(5)$. The mean number of branch points is significantly different on days $\mathrm{L} 3$ and $\mathrm{PO}(p<0.05,1$-tailed $t$-test $)$ and is not significantly different on day $\mathrm{P} 0$ and in mosaics ( $p>0.5,2$-tailed $t$-test).

represcntative drawings of afferents from the 3 regions of the $\mathrm{PH}$ array in a normal larva, and in a mosaic animal. The arbors of PH afferents from retained prolegs mapped appropriately within the neuropil and their structures resembled those of the larval controls. We conclude from these studies that the PH afferents on retained larval prolegs of heterochronic mosaic pupae retained their normal projections within the CNS.

\section{Morphology of PPR in heterochronic mosaics}

The morphometric technique used previously to quantify changes in PPR's structure analyzes the "dendritic density" of the neuron's contralateral arbor, revealed by cobalt backfilling the VNL (Weeks and Truman, 1985). Of the 7 motoneurons stained via the VNL, all remain ipsilateral except PPR, whose contralateral arbor can be viewed in isolation in the contralateral hemineuropil. The dendritic density score reflects the proportion of the contralateral hemineuropil containing PPR's dendrites. Figure $8, A, B$, shows representative VNL backfills made on days $\mathrm{L} 3$
Tahle 2. PPR's dendritic density in normally developing insects (A) and in heterochronic mosaic hemisegments (B)

Dendritic density $( \pm$ SEM)

\begin{tabular}{ll}
\hline A. Controls $(n)$ & \\
Day L3 (7) & $0.39 \pm 0.02^{b}$ \\
Day P0 (6) & $0.27 \pm 0.02^{b}$ \\
B. Heterochronic mosaics (n) & \\
Day P0 (5) & $0.28 \pm 0.03^{c}$
\end{tabular}

a Some data taken from Weeks (1987).

${ }^{b}$ Values are significantly different $(p<0.001)$.

c Value is significantly different from day L3 controls $(p<0.005)$ and is not significantly different from day P0 controls $(p>0.5)$.

All comparisons are 2-tailed $t$-tests.

and P0 in normal animals. PPR's contralateral arbor occupied less of the neuropil on day $P 0$, and the number of higher order branches was reduced (see also Weeks and Truman, 1985, 1986b; Weeks, 1987). PPR's dendritic density on days L3 and P0 was significantly different (Table $2 \mathrm{~A}$ ).

Even when applied locally, JHA in excessive amounts can interfere with pupal development throughout the body. Although our mosaics looked like normal pupae except for the retained proleg, it was important to establish that the JHA treatment did not have systemic effects, especially in the CNS. Because exposure of the CNS to JHA during the commitment pulse can rcduce or prevent PPR's subsequent regression (Weeks and Truman, 1986b), analysis of PPR's regression in the treated hemisegments provided a sensitive test for possible nonspecific effects of the JHA treatment. Accordingly, VNLs ipsilateral to retained prolegs were backfilled on day P0 $(n=5)$. The PPRs stained in this way appeared as regressed as untreated day $\mathrm{P} 0$ controls (Fig. 8, $B, C$ ), and their mean dendritic density did not differ significantly from that of day $\mathrm{P0}$ animals (Table 2B). Thus, there was no indication that the JHA applied to the epidermis interfered with normal pupal developmental of the CNS.

Synapses between the PH afferents and PPR do not occur in the dorsal, contralateral portion of PPR's arbor analyzed by the standard morphometric technique, but rather in the ventral ipsilateral neuropil to which PPR sends a small projection (Fig. 9A; Weeks and Jacobs, 1987; Peterson and Weeks, 1988). Thus, if JHA treatment or retention of the proleg interfered with the regression of PPR's ventral arbor, this result would have bcen missed in our standard analysis. It is not possible to visualize PPR's ventral arbor in VNL backfills because other motoneurons also project into this region (Weeks and Jacobs, 1987). Accordingly, PPRs associated with retained prolegs were stained by intracellular iontophoresis of cobalt on day $\mathrm{PO}(n=5)$. The dorsal, contralateral arbors of the stained PPRs appeared fully regressed (data not shown), consistent with the data obtained by backfilling (Fig. 8, Table 2B). The ventral portion of PPR's arbor in the mosaic hemisegments was reconstructed by computer, and compared to that in normal day $\mathrm{L} 3(n=7)$ and day P0 $(n=7)$ animals. Figure $9 B$ compares drawings of PPR's ventral arbor on day L3, day $\mathrm{P} 0$, and in a mosaic hemisegment on day $\mathrm{P} 0$; the arbors in the latter 2 cases were simpler than in larvae. A convenient morphometric parameter that reflects the complexity of a dendritic arbor is the number of branch points. Figure $9 C$ shows that the number of branch points in PPR's ventral arbor decreased significantly between days L3 and P0. 


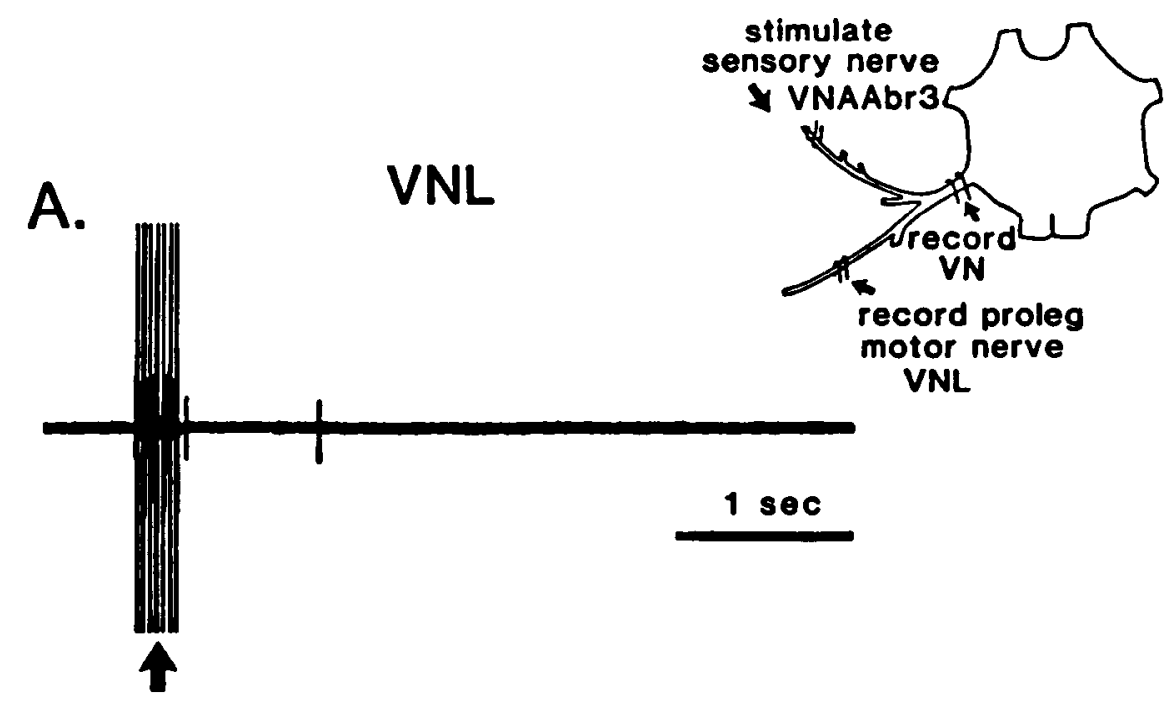

stim VNAAbr3

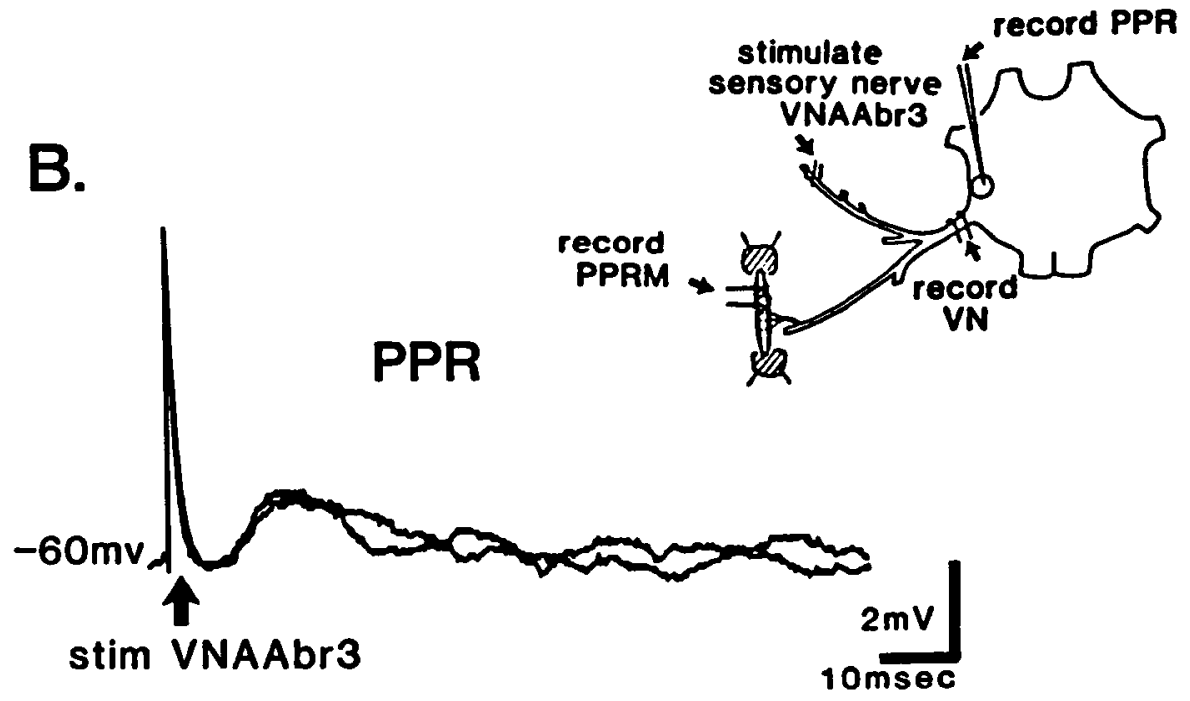

Figure 10. Electrophysiological recordings in heterochronic mosaic hemisegments. $A$, The inset shows the recording configuration (the same as in Fig. 2). VNAAbr3 was stimulated at 30 $\mathrm{Hz}$ for $500 \mathrm{msec}$ (the arrow marks the series of large stimulus artifacts) while monitoring $\mathrm{VN}$ en passant. Two motor spikes in VNL followed the stimulus train. $B$, The inset indicates the recording configuration (the same as in Fig. 3). Delivery of a single electrical shock to VNAAbr3 (arrow) while holding PPR at $-60 \mathrm{mV}$ evoked only a small synaptic depolarization. Two superimposed sweeps are shown.

Furthermore, the number of branch points in PPRs ipsilateral to a retaincd proleg did not differ significantly from the value in day P0 animals. We conclude that PPR regresses normally in mosaic hemisegments, even in the region of the arbor on which $\mathrm{PH}$ afferent synapses are made.

\section{Loss of the proleg withdrawal reflex in heterochronic mosaics}

Within the limitations of the anatomical techniques we used, these experiments provided evidence that the treated animals were mosaic at the neural level; i.e., the $\mathrm{PH}$ afferents were larval while PPR was pupal (regressed). To test the efficacy of the proleg withdrawal reflex in these animals we removed ganglia from mosaic segments on day P0 and made electrophysiological recordings.

Figure $10 \mathrm{~A}$ shows that a train of electrical stimuli applied to the PH sensory nerve evoked a minimal motor response in VNL of a mosaic hemisegment. The typical response consisted of a few or no spikes produced following the stimulus $(n=5)$. The finding that a few motoneuron spikes were sometimes produced (e.g., Fig. 10A) differs from the situation in normal day $\mathrm{P} 0$ animals (Fig. 2), but the motor response in mosaics was clearly reduced from that in the larval stage. More precise information was provided by intracellular recordings made on day $\mathrm{P} 0$ from PPRs ipsilateral to a retained proleg $(n=6)$. The mean $R_{\text {in }}$ of these motoneurons did not differ significantly from that measured in normal day P0 animals (Table 1, B, C). When single shocks were applied to VNAAbr3 while recording intracellularly from PPR in mosaic hemisegments, the evoked depolarization was small and invariably subthreshold (Fig. 10B). Table 1C shows that the mean afferent-evoked depolarization recorded with PPR held at $-60 \mathrm{mV}(3.0 \mathrm{mV})$ did not differ significantly from that recorded in normal day P0 animals $(3.7 \mathrm{mV})$.

These results show that the proleg withdrawal reflex was lost even in heterochronic mosaic animals in which the presynaptic $\mathrm{PH}$ afferents were maintained in the larval state. This finding suggests that the locus of change in the afferent-to-motoneuron pathway is postsynaptic, a likely factor being the regression of PPR's dendrites. 


\section{Discussion}

During metamorphosis, the Manduca nervous system controls sequentially a caterpillar, a pupa, and then an adult moth. Accordingly, at metamorphic molts, the neural circuits underlying outmoded behaviors must be dismantled and circuits for new behaviors assembled. In the present experiments we examined the factors that contribute to the elimination of a simple larval behavior, the proleg withdrawal reflex, that is rendered obsolete by the developmental loss of the prolegs at pupation. Our findings provide insight into the neural and endocrine mechanisms by which outmoded behaviors can be eliminated and provide a comparison for other studies that have identified factors important for the acquisition of new behaviors during metamorphosis.

\section{Neural factors underlying the developmental loss of the proleg withdrawal reflex}

Tactile stimulation of planta hairs, which is an effective stimulus for proleg withdrawal throughout larval life, no longer evokes withdrawal after day W2. Although alterations in peripheral structures (e.g., muscle degeneration) could contribute to this behavioral change, we found that the weakening of the synaptic pathways between $\mathrm{PH}$ afferents and proleg motoneurons in the CNS was sufficient to account for the loss of the behavior. The decreased effectiveness of the reflex pathway following the wandering stage was reflected in: (1) the gradual loss of the ability of electrical stimulation of PH afferent axons in VNAAbr3 to evoke proleg motoneuron firing (Fig. 2), and (2) the $78 \%$ decrease in the maximum synaptic depolarization evoked in PPR by stimulating VNAAbr3 (Fig. 3B; Table 1, A, B). In normal saline, VNAAbr3 stimulation evokes mono- and polysynaptic excitation of PPR as well as a polysynaptic inhibition that outlasts the excitation (Fig. 3B; Weeks and Jacobs, 1987). A developmental decrease in the size of the afferent-evoked depolarization could result from a change in the polysynaptic components, such as an increase in afferent-evoked inhibition. However, when recordings were made in high divalent saline to minimize or eliminate polysynaptic pathways, the size of the afferent-evoked depolarization still decreased significantly (by $68 \%$ ) between days $\mathrm{L} 3$ and P0 (Fig. 3C; Table 1, A, B). These results indicate that the monosynaptic component of the reflex weakens substantially, independent of any changes in the polysynaptic pathways. Although we did not investigate the afferentevoked polysynaptic effects in any detail, our data suggest that the polysynaptic depolarizing component may likewise weaken, and the hyperpolarizing component may be lost completely, between days L3 and P0 (Fig. 3).

Two mechanisms that could cause the size of the monosynaptic afferent-evoked depolarization recorded in PPR's cell body to decrease during development are: (1) the afferents could produce a smaller synaptic depolarization of PPR's dendrites on day P0 than on day L3, with the size of the depolarization recorded in the cell body at the 2 stages accurately reflecting the dendritic depolarization, or (2) the afferents could depolarize PPR's dendrites by the same amount on both days, but with the synaptic potentials on day PO being attenuated significantly before arriving at the recording site in the cell body. We favor mechanism (1), because it seems to be a reasonable electrophysiological correlate of the physical regression of PPR's dendrites, which could interrupt synaptic contacts with the afferents. Mechanism (2) is not supported by our finding that PPR's input resistance increased by $68 \%$ between days $\mathrm{L} 3$ and $\mathrm{P} 0$ (Table 1 , A, B); 2 factors that may contribute to this change are, first, a decrease in synaptic conductances due to a decrease in spontaneous synaptic inputs to PPR (Weeks and Truman, 1984a, b), and, second, changes in the electroanatomy of the motoneuron due to shrinkage of both the cell body and the dendritic tree (e.g., Rall, 1981). Both of these factors would be more likely to increase, rather than decrease, the size of the afferent-evoked depolarization recorded in PPR's cell body on day P0. However, even though $R_{\text {in }}$ increased, the decreased size of the afferentevoked potentials recorded in the cell body on day P0 could be caused by the appearance of a large resting conductance, or loss of active properties, in the dendritic region, which were not measurable from the cell body. Regardless of the exact mechanism(s) by which the monosynaptic pathway is weakened, the change is most likely attributable to postsynaptic factors because the synaptic potentials were reduced similarly in mosaic and in normal pupae (Table 1, B, C; see below).

We chose to study changes in synaptic inputs to PPR because of the great deal of background information available on its behavioral role, morphology, synaptic inputs, hormonal responses, and pharmacology. However, because PPR is fated to die on day $\mathrm{P} 2$, it is possible that the decreased strength of its afferent input at pupation might be related to the motoneuron's impending death. This possibility is lessened by the finding that the strength of PH inputs to other motoneurons that are not fated to die also decreases at pupation. For instance, all of the proleg motoneurons in VNL that are excited by VNAAbr 3 stimulation in larvae fail to respond by day P0 (Fig. 2), yet only a minority of them will subsequently die (Weeks and Ernst-Utzschneider, 1989). A specific example is provided by the proleg retractor motoneuron, accessory planta retractor (APR; Weeks and Truman, 1984b). All of the APRs regress by day P0 but only those in segments A5 and A6 die after pupation (Weeks and Ernst-Utzschneider, 1989). Intracellular recordings from the APRs in segments A3 and A4 show that the size of the depolarization evoked by VNAAbr 3 stimulation decreases between days $\mathrm{L} 3$ and $\mathrm{P} 0$ to an extent similar to that reportcd here for PPR (G. A. Jacobs, D. J. Sandstrom, and J. C. Weeks, unpublished observations). Thus, both motoneurons show a weakening of synaptic input from $\mathrm{PH}$ afferents as they regress, although only one motoneuron is programmed to subsequently die.

A striking finding was that the time course of the weakening of the afferent-to-motoneuron pathway parallels exactly the time course of PPR's dendritic regression. By our standard morphometric measurement, PPR remains in its larval form through day $\mathrm{WO}$, is first significantly regressed on day $\mathrm{W} 1$, and reaches maximum regression by day W3 (Weeks and Truman, 1985; Weeks, 1987). It remains in the fully regressed state until its death on day P2. Significantly, the ventral portion of PPR's arbor where sensory synapses are made also regresses between days L3 and P0 (Fig. 9). The excitation of proleg motoneurons including PPR by electrical stimulation of VNAAbr 3 was robust through day $\mathrm{W} 0$, weaker on days $\mathrm{W} 1$ and $\mathrm{W} 2$, and starting on day W3 was ineffective (Fig. 2). Thus, the extent of PPR's dendritic arbor and the strength of PPR's synaptic inputs from $\mathrm{PH}$ afferents declined coordinately over the final several days of larval life.

This temporal relationship suggested that the weakening of the monosynaptic afferent-to-motoneuron connections might be due to the regression of PPR's dendrites (e.g., due to physical 
interruption of the synaptic contacts). By this scenario, changes in the presynaptic afferents would not be necessary. A comparison of $\mathrm{PH}$ afferents on days L3 and $\mathrm{PO}$ suggested that their number and the density of their branching within the CNS (Fig. 5) may decrease slightly. Certainly their central projections were significantly reduced by day P2 (Fig. 5). Another possibility, which we did not examine, was whether the synaptic release properties of the afferents might also change; for instance, a decrease in the level of homosynaptic facilitation exhibited by the larval synapse (Weeks and Jacobs, 1987; B. A. Trimmer and J. C. Weeks, unpublished observations) could affect the ability of the afferents to excite motoneurons during stimulus trains such as in Figure 2. Due to these uncertainties about possible changes in anatomical or electrophysiological features of the PH afferents, it was difficult to assess how presynaptic changes might contribute to the weakening of the reflex during normal development.

To avoid this complication, we tested the role of presynaptic changes by generating heterochronic mosaic animals that had larval afferents and pupal motoneurons (Fig. 6A). The mosaic animals that were used for experiments bore normal-appearing PH arrays (Fig. 6B), each hair was associated with a $\mathrm{PH}$ afferent (Fig. 6C), and these afferents exhibited normal larval arbors within the CNS when examined individually (Fig. $7 \mathrm{C}$ ) or as a population (Fig. 7A). The $\mathrm{PH}$ afferents in mosaic animals thus appeared morphologically larval. In other studies of heterochronic mosaic pupae, Levine and collaborators (Levine et al., 1986; Levine, 1989) treated the peripheral cell bodies of body wall hair afferents with JHA during the commitment pulse of ecdysteroids in order to prevent the normal expansion of their arbors at pupation; this expansion is necessary for the afferents' ability to mediate a new, pupal-specific gin trap reflex (discussed below). These investigators found that the motor effects of afferents corresponded exactly with their morphology; JHA-treated afferents that remained in their larval form evoked larval like behaviors even though the CNS had become pupal, whereas control afferents with pupal arbors evoked pupal behaviors. Thus, the synaptic actions of the afferents were consistent with their morphology and developmental stage. In our experiments, therefore, we have assumed that the $\mathrm{PH}$ afferents on mosaic pupae retained the synaptic properties consistent with their larval morphology. The interpretation of our electrophysiological findings with mosaic animals rests on this critical, but untested, assumption.

When applied too liberally, JHA can exert systemic effects and interfere with pupal development throughout the body. Therefore, it was important to verify that the JHA treatment used to preserve a proleg did not interfere with PPR's regression. Two types of morphometric analysis verified that the PPRs in mosaic hemisegments regressed normally (Figs. 8, 9, Table 2). Most important, the ventral portion of PPR's arbor where the $\mathrm{PH}$ afferents make synaptic contact underwent normal regression. Thus, we assume that the afferent-to-motoneuron synapses were truly mosaic, with the prc- and postsynaptic ncurons being larval and pupal, respectively. The finding that the larval afferents did not interfere with the regression of PPR's ventral arbor (at least at the level of anatomical detail that we examined) is consistent with other studies suggesting that cellular interactions may not play a major role in the dendritic remodeling of motoneurons during metamorphosis in Manduca. For instance, the regression (Weeks and Truman, 1985) or expansion (Kent and Levine, 1988c) of motoneuron arbors can take place in the absence of contact with target muscles. These morphological changes are controlled by ecdysteroids and JH (see beginning of article), and all of the existing data are consistent with motoneurons being direct hormone targets (e.g., Weeks and Truman, 1986a; Weeks, 1987; Fahrbach and Truman, 1989; Griffin and Levine, 1989). If so, then the intrinsic programs activated in the motoneurons by developmental hormones may be relatively insensitive to cellular interactions. In contrast, cellular interactions have been shown to play an important role in the metamorphosis of sensory regions in the Manduca brain (e.g., Hildebrand et al., 1979; Maxwell and Hildebrand, 1981; Oland and Tolbert, 1987). In other insects, the presence or absence of afferent inputs during development can alter the shape of postsynaptic interneurons (e.g., Murphey et al., 1975; Hoy et al., 1985), but the possible involvement of hormones in these changes has not been explored.

The finding that the strength of the afferent-to-motoneuron pathway decreased in heterochronic mosaic hemisegments to the same extent as in normal pupae (Fig. 10, Table 1, B, C) suggests that postsynaptic changes are sufficient for the developmental loss of the proleg withdrawal reflex. An alternative finding, that the strength of the pathway remained strong in mosaics, would have implicated changes in the presynaptic afferents for the normal loss of the reflex. In larvae, the probability that a PH afferent produces a detectable EPSP in PPR, and the size of the EPSP when present, vary systematically across the PH array (Weeks and Jacobs, 1987; Levine and Weeks, 1989), a pattern that may be related to the somatotopic arrangement of PH afferent arbors within the CNS (Peterson and Weeks, 1988; Fig. 7C). The decreased magnitude of the afferent-evoked depolarization of PPR on day P0 could result from a decrease in the probability and/or size of the EPSP produced by each afferent; alternatively, some afferents could maintain their previous synaptic strengths while others disconnected completely. By our hypothesis that PPR's regression physically interrupts synaptic contacts, the balance between these 2 mechanisms could depend on the exact anatomical relationship between afferent boutons and motoneuron spines. For instance, preferential loss of distal branches in PPR's ventral arbor might cause the loss of connections made in that region, while inputs on the more proximal processes were maintained. In other systems, differences in the number of anatomical contacts made between preand postsynaptic neurons have been shown to correlate with the electrophysiological strength of the connection (Korn et al., 1982; Grantyn et al., 1984; Shepherd and Murphey, 1986). Because of our inability to record from or stain $\mathrm{PH}$ afferents on day $\mathrm{P0}$ we could not examine these possibilities directly, but comparable studies are feasible in mosaics, in which the retained larval afferents are experimentally accessible. These studies would provide more precise information about the relationship between anatomical contacts and synaptic strength.

An interesting comparison of the role of developmental changes in afferents is provided by the gin trap circuit in Manduca. The scgmental gin trap reflex of pupac (which mediates defensive closing of sensory pits) is mediated by a small population of body wall afferents that in the larva evoke mulitsegmental bending movements. The central arbors of these afferents expand during the larval-pupal transformation. To test the necessity of this growth for the appearance of the gin trap reflex, Levine and collaborators (Levine et al., 1986, 1989; Levine, 1989) produced heterochronic mosaic pupae in which presumptive gin trap afferents were kept larval. The central arbors of these afferents 
did not expand within the CNS and they were unable to evoke the pupal reflex, unlike adjacent untreated hairs. Thus, growth of the afferent arbors is necessary for the appearance of the gin trap reflex. Experiments with the opposite type of mosaic, in which the presumptive gin trap afferents were made pupal in an otherwise larval abdomen, showed that their growth was not sufficient to cause the switch to pupal behavior. By comparison, in the proleg system, the developmental status of the PH afferents appeared unrelated to whether the larval reflex was lost, suggesting that their pupal transformation was not necessary for the loss of the withdrawal reflex. In this case, changes in the postsynaptic cells (i.e., motoneurons) appear to be sufficient. These 2 examples illustrate that neurons at multiple hierarchical levels within the nervous system may participate in the developmental reorganization of a behavioral circuit. The difference in the roles played by sensory neurons in the gin trap and proleg circuits could relate to the fact that one behavior is being gained while the other is being lost; a determination of whether this correlation is a general one awaits studies of additional circuits.

\section{Relationship between changes in motoneuron structure and synaptic connections}

Other synaptic inputs to proleg motoneurons also decrease in strength during the larval-pupal transformation. For instance, the magnitude of the periodic waves of synaptic input that drive APR and PPR to fire rhythmic bursts of spikes during ecdysis (cuticle-shedding) motor patterns decreases substantially between the larval and pupal stages (Weeks and Truman, 1984b). In contrast, the synaptic inputs to other motoneurons that do not regress at pupation (Levine and Truman, 1985) are similar during larval and pupal ecdysis (Weeks and Truman, 1984a). This difference suggests that structural stability of a motoneuron during metamorphosis is associated with the conservation of synaptic inputs, whereas dendritic regression is associated with the weakening of inputs. Dendritic regression does not necessarily cause all inputs to be weakened, however, as is illustrated by motoneuron APR. In the larval stage, APR receives monosynaptic inputs from a number of identified interneurons (Sandstrom and Weeks, 1988, and unpublished observation; Levine and Weeks, 1989). On day P0, some of these interneurons produce synaptic potentials in APR that are the same size, or larger, than they were on day L3; over the same time period, the inputs from $\mathrm{PH}$ afferents get weaker. Such patterns of shifting synaptic strengths may reflect the functional respecification process, as a motoneuron is removed from old behavioral circuits and begins to participate in new ones. At the anatomical level, the key to how the strength of the connections changes during development may depend critically on where the synapses are made on the motoneuron arbor; for instance, those made on distal processes may be more likely to be lost during regression than those on more proximal processes.

Of course, not all changes in synaptic connections involve obvious changes in motoneuron morphology. In Manduca, factors that may influence synaptic signalling without requiring morphological changes include alterations in the current/voltage properties of motoneurons, activity-dependent synaptic properties (e.g., facilitation), synaptically induced alterations in motoneuron spike threshold, and peptide modulation of synaptic connections (Levine and Truman, 1983; Weeks and Jacobs, 1987; Trimmer and Weeks, 1989; Waldrop and Levine, 1989). Our relatively crude morphological measurements of motoneuron structure would miss any subtle alterations, which can have strong effects on synaptic transmission (e.g., Rall, 1970, 1974; Miller et al., 1985). Even when morphological changes are apparent, they do not necessarily correspond to changes in synaptic connectivity; for instance, between the larval and pupal stages, the strength of synaptic drive to certain motoneurons during preecdysis (cuticle loosening) motor patterns decreases in an anterior-to-posterior segmental gradient, yet these motoneurons regress most extensively in the anterior ganglia (Miles and Weeks, 1988, and unpublished observations; Weeks et al., 1989).

Although many factors undoubtedly contribute to the reorganization of neural circuits during metamorphosis, our studies of proleg motoneurons suggest that one mechanism that may contribute to the elimination of outmoded bchaviors is the ccdysteroid-triggered regression of motoneuron dendrites. Other classes of motoneurons in addition to proleg motoneurons also regress structurally at the end of larval life or early in the pupal stage, which could disconnect them from their respective behavioral circuits. These include motoneurons that innervate a variety of types of abdominal body wall muscles, and muscles in the larval thoracic legs (Truman and Reiss, 1976, 1988; Levine and Truman, 1985; Kent and Levine, 1988c; Weeks and Ernst-Utzschneider, 1989; Miles and Weeks, unpublished observations). This report, however, is the first to correlate the regression of motoneuron dendrites with the developmental loss of a specific behavior.

Regardless of whether or not a motoneuron regresses at pupation, most show dendritic growth during adult development. The new growth is correlated with the acquisition of new synaptic inputs and participation in new behavioral circuits. For instance, the sign of postural reflexes evoked by abdominal stretch receptor neurons reverses after the pupa emerges as a moth; this change is associated with the ecdysteroid-triggered growth of motoneuron arbors across the ganglionic midline, which allows them to receive new, monosynaptic excitatory inputs from contralateral stretch receptor neurons (Levine and Truman, 1982). The motoneurons that innervate the larval thoracic legs in Manduca regress massively at pupation, followed by dendritic growth into neuropil regions containing afferent terminations from the new adult legs (Kent and Levine, 1988a, b, c); this arrangement presumably promotes the development of circuits mediating new segmental leg reflexes. The relationship between dendritic growth and the formation of new synaptic connections during metamorphosis is also illustrated by sensory neurons in the gin trap circuit (Levine et al., 1986, 1989; Levine, 1989).

Thus, a general mechanism that contributes to the appearance or loss of behaviors during metamorphosis is the expansion or regression of motoneuron arbors. This relationship is particularly apparent in larval motoneurons that are respecified to innervate new adult muscles. A common pattern is the regression of neuronal processes in the late larval or early pupal stage, which contributes to the elimination of outmoded larval inputs. At the same time, or as new adult-specific processes bcgin to grow, synaptic inputs appropriate for pupal or adult behavior increase in strength, or form de novo. The developmental hormones, juvenile hormone and the ecdysteroids, play a pivotal role in these events by stimulating the morphological changes in neurons (e.g., Weeks and Truman, 1985, 1986a, b; Levine et al., 1986; Weeks, 1987; Truman and Reiss, 1988; Levine, 1989; Weeks and Ernst-Utzschneider, 1989). This relationship between hormones and neural circuits has parallels in the vertehrate nervous system. For instance, in birds (Nottebohm, 1981) and rodents (Forger and Breedlove, 1987) that breed seasonally, 
the dendritic arbors of neurons involved in male sexual behavior expand or regress in response to the periodic changes in blood levels of gonadal steroid hormones. Hormonally mediated dendritic regression in these neurons dismantles the circuits underlying sexual behavior, which remain nonfunctional until neuronal reexpansion is initiatcd in response to hormonal cues at the next breeding season. In these examples and during metamorphosis, steroid-mediated structural changes in neurons play a key role in the periodic construction and dismantling of behavioral circuits throughout postembryonic life.

\section{References}

Bacon, J. P., and J. S. Altman (1977) A silver intensification method for cobalt filled neurons in whole mount preparations. Brain Res. 138: 359-363.

Bell, R. A., and F. A. Joachim (1976) Techniques for rearing laboratory colonies of tobacco hornworms and pink bollworms. Ann. Entomol. Soc. Am. 69: 365-373.

Booker, R., and J. W. Truman (1987a) Postembryonic neurogenesis in the CNS of the tobacco hornworm, Manduca sexta. I. Neuroblast arrays and the fate of their progeny during metamorphosis. J. Comp. Neurol. 255: 548-559.

Booker, R., and J. W. Truman (1987b) Postembryonic neurogenesis in the CNS of the tobacco hornworm, Manduca sexta. II. Hormonal control of imaginal nest cell degeneration and differentiation during metamorphosis. J. Neurosci. 7: 4107-4114.

Brogan, R. T., and R. M. Pitman (1981) Axonal regeneration in an identified insect motoneurone. J. Physiol. (Lond.) 285: 231-255.

Cohen, J. L., K. R. Weiss, and I. Kupfermann (1978) I. Motor control of buccal muscles in Aplysia. J. Neurophysiol. 41: 157-180.

Copenhaver, P. F., and J. W. Truman (1982) The role of eclosion hormone in the larval ecdyses of Manduca sexta. J. Insect Physiol. 28: 695-701.

Fahrbach, S. E., and J. W. Truman (1989) Autoradiographic identification of ecdysteroid binding cells in the nervous system of the moth Manduca sexta. J. Neurobiol. 20: 681-702.

Fain, M. J., and L. M. Riddiford (1977) Requirements for molting of the crochet epidermis of the tobacco hornworm larva in vivo and in vitro. Wilhelm Roux Arch. EntwMech. Org. 181: 285-307.

Forger, N. G., and S. M. Breedlove (1987) Seasonal variation in mammalian striated muscle mass and motoneuron morphology. J. Neurobiol. 18: 155-165.

Giebultowicz, J. M., and J. W. Truman (1984) Sexual differentiation in the terminal ganglion of the moth Manduca sexta: Role of sexspecific neuronal death. J. Comp. Neurol. 226: 87-95.

Grantyn, R., A. I. Shapolalov, and B. I. Shiriaev (1984) Relation between structural and release parameters at the frog sensory-to-motor synapse. J. Physiol. (Lond.) 349: 459-474.

Griffin, L., and Levine, R. B. (1989) Hormonal influences on insect motoneurons developing in cell culture. Soc. Neurosci. Abstr. 15: 87.

Hildebrand, J. G., L. M. Hall, and B. C. Osmond (1979) Distribution of binding sites for 125-I-labelled $\alpha$-bungarotoxin in normal and deafferented antennal lobes of Manduca sexta. Proc. Natl. Acad. Sci. USA 76: 499-503.

Hoy, R. R., T. G. Nolen, and G. C. Casaday (1985) Dendritic sprouting and compensatory synaptogenesis in an identified interneuron following auditory deprivation in a cricket. Proc. Natl. Acad. Sci. USA 82: 7772-7776.

Jacobs, G. A., and J. C. Weeks (1986) Developmental disappearance of a monosynaptic sensory-to-motor reflex in the tobacco hornworm, Manduca sexta. Soc. Neurosci. Abstr. 12: 1165.

Kent, K. S., and R. B. Levine (1988a) Neural control of leg movements in a metamorphic insect: Sensory and motor elements of the larval thoracic legs in Manduca sexta. J. Comp. Neurol. 271: 559-576.

Kent, K. S., and R. B. Levine (1988b) Neural control of leg movements in a metamorphic insect: Persistence of the larval leg motor neurons to innervate the adult legs of Manduca sexta. J. Comp. Ncurol. 276: $30-43$.

Kent, K. S., and R. B. Levine (1988c) Reorganization of an identified leg motor neuron during metamorphosis of the moth Manduca sexta. Soc. Neurosci. Abstr. 14: 1004.
Korn, H., A. Mallet, A. Triller, and D. S. Faber (1982) Transmission at a central inhibitory synapse. II. Quantal description of release, with a physical correlate for binomial $n$. J. Neurophysiol. 48: 679-707.

Levine, R. B. (1989) Expansion of the central arborizations of persistent sensory neurons during insect metamorphosis: The role of the steroid hormone, 20-hydroxyecdysone. J. Neurosci. 9: 1045-1054.

Levine, R. B., and J. W. Truman (1982) Metamorphosis of the insect nervous system: Changes in morphology and synaptic interactions of identified neurons. Nature 299: 250-252.

Levine, R. B., and J. W. Truman (1983) Peptide activation of a simple neural circuit. Brain Res. 279: 335-338.

Levine, R. B., and J. W. Truman (1985) Dendritic reorganization of abdominal motoneurons during metamorphosis of the moth Manduca sexta. J. Neurosci. 5: 2424--2431.

Levine, R. B., and J. C. Weeks (1989) Reorganization of neural circuits and behavior during insect metamorphosis. In Perspectives on Neural Systems and Behavior, T. J. Carew and D. Kelley, eds., pp. 195-228, Liss, New York.

Levine, R. B., J. W. Truman, D. Linn, and C. M. Bate (1986) Endocrine regulation of the form and function of axonal arbors during insect metamorphosis. J. Neurosci. 6: 293-299.

Levine, R. B., B. Waldrop, and D. Tamarkin (1989) The use of hormonally-induced mosaics to study alterations in the synaptic connections made by persistent sensory neurons during insect metamorphosis. J. Neurobiol. 20: 326-338.

Maxwell, G. D., and J. G. Hildebrand (1981) Anatomical and neurochemical consequences of deafferentation in the development of the visual system of the moth Manduca sexta. J. Comp. Neurol. 195: 667-680.

Miles, C. I., and J. C. Weeks (1988) Developmental changes in preecdysis motor patterns of the moth, Manduca sexta. Soc. Neurosci. Abstr. 14: 998.

Miller, J. P., W. Rall, and J. Rinzel (1985) Synaptic amplification in dendritic spines. Brain Res. 325: 325-330.

Miyazaki, S. (1980) The ionic mechanism of action potentials in neurosecretory cells and nonneurosecretory cells of the silkworm. J. Comp. Physiol. 140: 43-52.

Murphey, R. K., B. Mendenhall, J. Palka, and J. S. Edwards (1975) Deafferentation slows the growth of specific dendrites of identified giant interneurons. J. Comp. Neurol. 159: 407-418.

Nevin, R., J. W. Tromp, J. P. Miller, and G. A. Jacobs (1988) Electroanatomical parameters of sensory interneurons in the cricket cercal sensory system. Soc. Neurosci. Abstr. 14: 379.

Nottebohm, F. (1981) A brain for all seasons: Cyclical anatomical changes in song control nuclei of the canary brain. Science 214: 13681370 .

Oland, L. A., and L. P. Tolbert (1987) Glial patterns during early development of antennal lobes of Manduca sexta: A comparison between normal lobes and lobes deprived of antennal axons. J. Comp. Neurol. 255: 196-207.

Peterson, B. A., and J. C. Weeks (1988) Somatotopic mapping of sensory neurons innervating mechanosensory hairs on the larval prolegs of Manduca sexta. J. Comp. Neurol. 275: 128-144.

Rall, W. (1970) Cable properties of dendrites and effects of synaptic location. In Excitatory Synaptic Mechanisms, P. Anderson and J. K. S. Jansen, eds., pp. 175-187, Universitetsforlaget, Oslo, Norway.

Rall, W. (1974) Dendritic spines, synaptic potency and neuronal plasticity. In Cellular Mechanisms Subserving Changes in Neuronal Activity, Brain Inf. Serv. Rpt. no. 3, C. D. Woody, K. A. Brown, T. J. Crow, and J. D. Knispal, eds., pp. 13-21, UCLA Press, Los Angeles, CA.

Rall, W. (1981) Functional aspects of neuronal geometry. In Neurones Without Impulses, A. Roberts and B. M. H. Bush, eds., pp. 203-209, Cambridge U. P., Cambridge.

Riddiford, L. M. (1985) Hormone action at the cellular level. In Comprehensive Insect Physiology, Biochemistry, and Pharmacology, G. A. Kerkut and L. I. Gilbert, eds., Vol. 8, pp. 37-84, Pergamon, New York.

Sandstrom, D. J., and J. C. Weeks (1988) Identified interneurons in larval and pupal abdominal ganglia of the tobacco hornworm, Manduca sexta. Soc. Neurosci. Abstr. 14: 1003.

Shepherd, D., and R. K. Murphey (1986) Competition regulates the efficacy of an identified synapse in crickets. J. Neurosci. 6: 31523160 .

Taylor, H. M., and J. W. Truman (1974) Metamorphosis of the ab- 
dominal ganglia of the tobacco hornworm, Manduca sexta. J. Comp. Physiol. 90: 367-388.

Trimmer, B. A., and J. C. Weeks (1989) Effects of nicotinic and muscarinic agents on an identified motoneurone and its direct afferent inputs in larval Manduca sexta. J. Exp. Biol. 144: 303-337.

Truman, J. W. (1976) Development and hormonal release of adult bchavior patterns in silkmoths. J. Comp. Physiol. 107: 39-48.

Truman, J. W. (1983) Programmed cell death in the nervous system of an adult insect. J. Comp. Neurol. 216: 445-452.

Truman, J. W. (1989) Hormonal approaches for studying nervous system development in insects. Adv. Insect Physiol. 21: 1-34.

Truman, J. W., and S. E. Reiss (1976) Dendritic reorganization of an identified motoneuron during metamorphosis of the tobacco hornworm, Manduca sexta. Science 192: 477-479.

Truman, J. W., and S. E. Reiss (1988) Hormonal regulation of the shape of identified motoneurons in Manduca sexta. J. Neurosci. 8. $765-775$.

Truman, J. W., and L. M. Schwartz (1984) Steroid regulation of neuronal death of the moth nervous system. J. Neurosci. 4: 274-280.

Waldrop, B., and R. B. Levine (1989) Development of the gin trap reflex in Manduca sexta: A comparison of larval and pupal motor responses. J. Comp. Physiol. 165: 743-753.

Weeks, J. C. (1987) Time course of hormonal independence for developmental events in neurons and other cell types during insect metamorphosis. Dev. Biol. 124: 163-176.

Weeks, J. C., and K. Ernst-Utzschneider (1989) Respecification of larval proleg motoneurons during metamorphosis of the tobacco hornworm, Manduca sexta: Segmental dependence and hormonal regulation. J. Neurobiol. 20: 569-592.

Weeks, J. C., and G. A. Jacobs (1987) A reflex behavior mediated by monosynaptic connections between hair afferents and motoneurons in the larval tobacco hornworm, Manduca sexta. J. Comp. Physiol. 160: 315-329.
Weeks, J. C., and G. A. Jacobs (1988) Developmental changes in postsynaptic neurons cause loss of a monosynaptic reflex during metamorphosis in Manduca sexta. Soc. Neurosci. Abstr. 14: 998.

Weeks, J. C., and R. B. Levine (1990) Postembryonic neuronal plasticity and its hormonal control during insect metamorphosis. Annu. Rev. Neurosci. 13: 183-194.

Weeks, J. C., and J. W. Truman (1984a) Neural organization of peptide-activated ecdysis behaviors during the metamorphosis of Manduca sexta. I. Conservation of the peristalsis motor pattern at the larval-pupal transformation. J. Comp. Physiol. 155: 407-422.

Weeks, J. C., and J. W. Truman (1984b) Neural organization of peptide-activated ecdysis behaviors during the metamorphosis of Manduca sexta. II. Retention of the proleg motor pattern despite loss of the prolegs at pupation. J. Comp. Physiol. 155: 423-433.

Weeks, J. C., and J. W. Truman (1985) Independent steroid control of the fates of motoneurons and their muscles during insect metamorphosis. J. Neurosci. 5: 2290-2300.

Weeks, J. C., and J. W. Truman (1986a) Steroid control of neuron and muscle development during the metamorphosis of an insect. J. Neurobiol. 17: 249-267.

Wecks, J. C., and J. W. Truman (1986b) Hormonally mediated rcprogramming of muscles and motoneurons during the larval-pupal transformation of the tobacco hornworm, Manduca sexta. J. Exp. Biol. 125: 1-13.

Weeks, J. C., G. A. Jacobs, and C. I. Miles (1989) Hormonally-mediated modifications of neuronal structure, synaptic connectivity, and behavior during metamorphosis of the tobacco hornworm, Manduca sexta. Am. Zoologist 29: 1331-1334.

Wolfgang, W., and L. M. Riddiford (1987) Cuticular mechanics during larval development of the tobacco hornworm, Manduca sexta. J. Exp. Biol. 128: 19-33. 